\title{
The Kinetics of Addition and Fragmentation in Reversible Addition Fragmentation Chain Transfer (RAFT) Polymerization: An Ab Initio Study
}

Michelle L. Coote*

Research School of Chemistry, Australian National University, Canberra, ACT 0200, Australia

\section{SUPPORTING INFORMATION}

(Tables S1-S4, Figures S1-S4, Total 18 pages)

\footnotetext{
* To whom correspondence should be addressed, email: mcoote@rsc.anu.edu.au
} 
Table S1. GAUSSIAN Archive Entries for B3-LYP/6-31G(d) Optimized Geometries*

\begin{abstract}
Formal TS: $\cdot \mathrm{CH}_{3}+\mathrm{S}=\mathrm{C}\left(\mathrm{CH}_{3}\right) \mathrm{SCH}_{3}(1)$
$1 \backslash 1 \backslash G I N C-S C 70 \backslash F T S \backslash U B 3 L Y P \backslash 6-31 G(d) \backslash C 4 H 9 S 2(2) \backslash M L C 501 \backslash 17-J u l-2002 \backslash 1 \backslash \backslash \# N \quad U$ B3LYP $/ 6-31 \mathrm{G}(\mathrm{D})$ OPT $=(\mathrm{TS}, \mathrm{EF}, \mathrm{CALCFC}, \mathrm{Z}-\mathrm{MATRIX}, \mathrm{MAXCYC}=100)$ FREQ MAXDISK $=131$ $072000 \mathrm{SCF}=(\mathrm{TIGHT}, \mathrm{MAXCYC}=100) \backslash \backslash$ mescmesmeTS $-\mathrm{C} 1 \mathrm{~b}-6$ dub $3 \backslash \backslash 0,2 \backslash \mathrm{S} \backslash \mathrm{C}, 1, \mathrm{~B} 1 \backslash \mathrm{S}, 2$

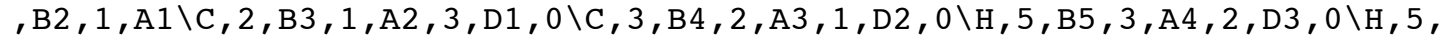
$\mathrm{B} 6,3, \mathrm{~A} 5,6, \mathrm{D} 4,0 \backslash \mathrm{H}, 5, \mathrm{~B} 7,3, \mathrm{~A} 6,6, \mathrm{D} 5,0 \backslash \mathrm{C}, 1, \mathrm{~B} 8,2, \mathrm{~A} 7,3, \mathrm{D} 6,0 \backslash \mathrm{H}, 9, \mathrm{~B} 9,1, \mathrm{~A} 8,2$, D7, $0 \backslash \mathrm{H}, 9, \mathrm{~B} 10,1, \mathrm{~A} 9,10, \mathrm{D} 8,0 \backslash \mathrm{H}, 9, \mathrm{~B} 11,1, \mathrm{~A} 10,10, \mathrm{D} 9,0 \backslash \mathrm{H}, 4, \mathrm{~B} 12,2, \mathrm{~A} 11,1, \mathrm{D} 10,0 \backslash \mathrm{H}, 4$ , $\mathrm{B} 13,2, \mathrm{~A} 12,1, \mathrm{D} 11,0 \backslash \mathrm{H}, 4, \mathrm{~B} 14,2, \mathrm{~A} 13,1, \mathrm{D} 12,0 \backslash \backslash \mathrm{B} 1=1.65090491 \backslash \mathrm{B} 2=1.76226163 \backslash$ $B 3=1.51367224 \backslash B 4=1.81883498 \backslash B 5=1.09315991 \backslash B 6=1.09202887 \backslash B 7=1.09173616 \backslash$ $B 9=1.08242665 \backslash B 10=1.08312635 \backslash B 11=1.08306075 \backslash B 12=1.09253826 \backslash B 13=1.09797$ $114 \backslash \mathrm{B} 14=1.09596882 \backslash \mathrm{A} 1=125.62145537 \backslash \mathrm{A} 2=122.8978683 \backslash \mathrm{A} 3=103.82801251 \backslash \mathrm{A} 4=1$ $06.14424305 \backslash \mathrm{A} 5=110.34937531 \backslash \mathrm{A} 6=110.58546476 \backslash \mathrm{A} 7=112.97083096 \backslash \mathrm{A} 8=96.1395$ $3096 \backslash \mathrm{A} 9=98.03637008 \backslash \mathrm{A} 10=92.94745782 \backslash \mathrm{A} 11=109.96652475 \backslash \mathrm{A} 12=109.5126919 \backslash \mathrm{A}$ $13=112.36453295 \backslash D 1=-183.88973235 \backslash D 2=-1.2889061 \backslash D 3=180.94499783 \backslash D 4=119$. $40180162 \backslash D 5=-119.54246872 \backslash D 6=-93.40650499 \backslash D 7=-86.24438242 \backslash D 8=120.62612$ $263 \backslash D 9=-119.6656341 \backslash D 10=34.13325402 \backslash D 11=-84.13835705 \backslash D 12=156.15698465 \backslash$ $\mathrm{B} 8=2.76574916 \backslash \backslash$ Version=DEC-AXP-OSF $/ 1-\mathrm{G} 98 \mathrm{RevA} .11 .3 \backslash \mathrm{HF}=-954.1441368 \backslash \mathrm{S} 2=0$ $.772159 \backslash \mathrm{S} 2-1=0 . \backslash \mathrm{S} 2 \mathrm{~A}=0.75014 \backslash \mathrm{RMSD}=8.680 \mathrm{e}-09 \backslash \mathrm{RMSF}=1.475 \mathrm{e}-05 \backslash \mathrm{Dipole}=0.278$ $789,0.2862317,0.3519356 \backslash P G=\mathrm{C} 01 \quad[\mathrm{X}(\mathrm{C} 4 \mathrm{H} 9 \mathrm{~S} 2)] \backslash \backslash @$
\end{abstract}

\title{
Variational TS: $\cdot \mathrm{CH}_{3}+\mathrm{S}=\mathrm{C}\left(\mathrm{CH}_{3}\right) \mathrm{SCH}_{3}(1)$
}

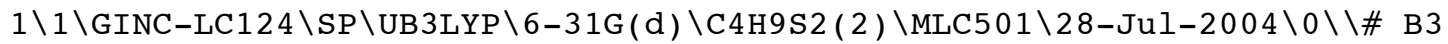
LYP /6-31G* SCF=TIGHT MAXDISK=402653184 \\CVT TS $\mathrm{Z}=\mathrm{Me} \mathrm{R}=\mathrm{Me} \backslash \backslash 0,2 \backslash S, 0,1.12$ $0491,-0.087753,-0.810554 \backslash \mathrm{C}, 0,-0.186781,0.73548,-0.162051 \backslash \mathrm{S}, 0,-1.711746$ $, 0.00266,0.336267 \backslash \mathrm{C}, 0,-0.143844,2.211067,0.16196 \backslash \mathrm{C}, 0,-1.526348,-1.7526$ $81,-0.105148 \backslash \mathrm{H}, 0,-2.463287,-2.237714,0.181239 \backslash \mathrm{H}, 0,-1.36145,-1.86165,-1$ $.179059 \backslash \mathrm{H}, 0,-0.691583,-2.201652,0.436377 \backslash \mathrm{C}, 0,2.783219,-0.785248,0.8479$ $1 \backslash \mathrm{H}, 0,3.402121,0.101529,0.932208 \backslash \mathrm{H}, 0,2.179498,-1.041046,1.712991 \backslash \mathrm{H}, 0,3$ $.202745,-1.617971,0.291688 \backslash \mathrm{H}, 0,0.465206,2.739893,-0.576144 \backslash \mathrm{H}, 0,0.31407$ $3,2.370587,1.148178 \backslash \mathrm{H}, 0,-1.144728,2.657803,0.18509 \backslash \backslash$ Version=x86-LinuxG03RevB.03 $\backslash$ State $=2-A \backslash H F=-954.1469672 \backslash \mathrm{S} 2=0.787483 \backslash \mathrm{S} 2-1=0 . \backslash \mathrm{S} 2 \mathrm{~A}=0.750218 \backslash$ RMSD $=6.727 e-09 \backslash \mathrm{Dipole}=-0.0741845,-0.2183756,0.4647843 \backslash \mathrm{PG}=\mathrm{C} 01 \quad[\mathrm{X}(\mathrm{C} 4 \mathrm{H} 9 \mathrm{~S} 2$ )$] \backslash \$

\section{Formal TS: $\cdot \mathrm{CH}_{3}+\mathrm{S}=\mathrm{C}(\mathrm{Ph}) \mathrm{SCH}_{3}(2)$}

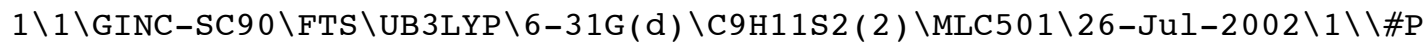
B3LYP / 6-31G* OPT $=(T S, Z-M A T R I X, M A X C Y C=100$, CALCFC , NOEIGENTEST $)$ MAXDISK=1 $31072000 \mathrm{SCF}=(\mathrm{QC}, \mathrm{MAXCYC}=100) \mathrm{FREQ} \backslash \backslash \mathrm{TS}$ for $\mathrm{CH} 3+\mathrm{RAFT}$ agent $\mathrm{S}=\mathrm{C}(\mathrm{Ph})-\mathrm{S}-\mathrm{CH}$ 3 ( TS: $\mathrm{Z}=\mathrm{ph}, \mathrm{R}=\mathrm{me})(\mathrm{a}) \backslash \backslash 0,2 \backslash \mathrm{C} \backslash \mathrm{S}, 1, \mathrm{~B} 1 \backslash \mathrm{S}, 1, \mathrm{~B} 2,2, \mathrm{~A} 1 \backslash \mathrm{C}, 3, \mathrm{~B} 3,1, \mathrm{~A} 2,2, \mathrm{D} 1,0 \backslash \mathrm{H}, 4$, $\mathrm{B} 4,3, \mathrm{~A} 3,1, \mathrm{D} 2,0 \backslash \mathrm{H}, 4, \mathrm{~B} 5,3, \mathrm{~A} 4,5, \mathrm{D} 3,0 \backslash \mathrm{H}, 4, \mathrm{~B} 6,3, \mathrm{~A} 5,5, \mathrm{D} 4,0 \backslash \mathrm{C}, 1, \mathrm{~B} 7,2, \mathrm{~A} 6,3, \mathrm{D} 5$, $0 \backslash \mathrm{C}, 8, \mathrm{~B} 8,1, \mathrm{~A} 7,2, \mathrm{D} 6,0 \backslash \mathrm{C}, 9, \mathrm{~B} 9,8, \mathrm{~A} 8,1, \mathrm{D} 7,0 \backslash \mathrm{C}, 10, \mathrm{~B} 10,9, \mathrm{~A} 9,8, \mathrm{D} 8,0 \backslash \mathrm{C}, 11, \mathrm{~B} 11$, $10, \mathrm{~A} 10,9, \mathrm{D} 9,0 \backslash \mathrm{C}, 12, \mathrm{~B} 12,11, \mathrm{~A} 11,10, \mathrm{D} 10,0 \backslash \mathrm{H}, 13, \mathrm{~B} 13,12, \mathrm{~A} 12,11, \mathrm{D} 11,0 \backslash \mathrm{H}, 12, \mathrm{~B}$ $14,11, \mathrm{~A} 13,13, \mathrm{D} 12,0 \backslash \mathrm{H}, 11, \mathrm{~B} 15,10, \mathrm{~A} 14,12, \mathrm{D} 13,0 \backslash \mathrm{H}, 10, \mathrm{~B} 16,9, \mathrm{~A} 15,11, \mathrm{D} 14,0 \backslash \mathrm{H}$, 9 , $\mathrm{B} 17,8, \mathrm{~A} 16,10, \mathrm{D} 15,0 \backslash \mathrm{C}, 2, \mathrm{~B} 18,1, \mathrm{~A} 17,3, \mathrm{D} 16,0 \backslash \mathrm{H}, 19, \mathrm{~B} 19,2, \mathrm{~A} 18,1, \mathrm{D} 17,0 \backslash \mathrm{H}, 19$ , B20 , $2, \mathrm{~A} 19,1, \mathrm{D} 18,0 \backslash \mathrm{H}, 19, \mathrm{~B} 21,2, \mathrm{~A} 20,1, \mathrm{D} 19,0 \backslash \backslash \mathrm{B} 18=2.85785244 \backslash \mathrm{A} 17=112.8854$ $7308 \backslash \mathrm{D} 16=87.54234418 \backslash \mathrm{B} 19=1.08281955 \backslash \mathrm{B} 20=1.08254509 \backslash \mathrm{B} 21=1.08254817 \backslash \mathrm{A} 18=$ $96.34150121 \backslash A 19=93.33447677 \backslash A 20=94.2496342 \backslash D 17=-10.5489024 \backslash D 18=-130.55$ $97207 \backslash \mathrm{D} 19=109.66341591 \backslash \mathrm{B} 1=1.65766937 \backslash \mathrm{B} 2=1.77315841 \backslash \mathrm{B} 3=1.81803301 \backslash \mathrm{B} 4=1$. $09350034 \backslash B 5=1.09209587 \backslash B 6=1.09142411 \backslash B 7=1.48423492 \backslash B 8=1.40872449 \backslash B 9=1$. $3934021 \backslash \mathrm{B} 10=1.39544748 \backslash \mathrm{B} 11=1.39759958 \backslash \mathrm{B} 12=1.39092175 \backslash \mathrm{B} 13=1.08452426 \backslash \mathrm{B} 1$ $4=1.08664308 \backslash \mathrm{B} 15=1.0866489 \backslash \mathrm{B} 16=1.08655345 \backslash \mathrm{B} 17=1.08514617 \backslash \mathrm{A} 1=123.660962$

\footnotetext{
* The B3-LYP/6-31G(d) geometries of the reactants and products are provided in the Supporting Information of
} Coote, M. L. Macromolecules 2004, 37, 5023-5031. 
$99 \backslash \mathrm{A} 2=103.49634073 \backslash \mathrm{A} 3=105.71669512 \backslash \mathrm{A} 4=110.60606647 \backslash \mathrm{A} 5=110.70344078 \backslash \mathrm{A} 6=$ $123.44769264 \backslash A 7=121.77261104 \backslash A 8=120.70458187 \backslash A 9=120.28905352 \backslash A 10=119.5$ $8591948 \backslash A 11=120.32573329 \backslash A 12=120.46930469 \backslash A 13=120.08894967 \backslash A 14=120.202$ $94711 \backslash A 15=119.53022621 \backslash A 16=119.83190111 \backslash D 1=7.65849732 \backslash D 2=177.95770467 \backslash$ $\mathrm{D} 3=-119.30931244 \backslash \mathrm{D} 4=119.36346225 \backslash \mathrm{D} 5=182.6028638 \backslash \mathrm{D} 6=209.56180499 \backslash \mathrm{D} 7=179$ $.85020311 \backslash \mathrm{D} 8=0.41205611 \backslash \mathrm{D} 9=-0.43641868 \backslash \mathrm{D} 10=-0.05221285 \backslash \mathrm{D} 11=180.0617337$ $2 \backslash D 12=179.91322301 \backslash D 13=180.26246934 \backslash D 14=180.47708809 \backslash D 15=181.99245773 \backslash$ $\backslash$ Version=DEC-AXP-OSF $/ 1-G 98 R e v A .11 .3 \backslash \mathrm{HF}=-1145.8826857 \backslash \mathrm{S} 2=0.77023 \backslash \mathrm{S} 2-1=0$ $. \backslash \mathrm{S} 2 \mathrm{~A}=0.75015 \backslash \mathrm{RMSD}=0.000 \mathrm{e}+00 \backslash \mathrm{RMSF}=2.289 \mathrm{e}-05 \backslash \mathrm{Dipole}=0.0939665,0.1867077$ ,$-0.4556698 \backslash \mathrm{PG}=\mathrm{C} 01 \quad[\mathrm{X}(\mathrm{C} 9 \mathrm{H} 11 \mathrm{~S} 2)] \backslash \backslash @$

\section{Variational TS: $\cdot \mathrm{CH}_{3}+\mathrm{S}=\mathrm{C}(\mathrm{Ph}) \mathrm{SCH}_{3}(2)$}

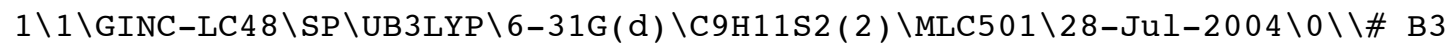

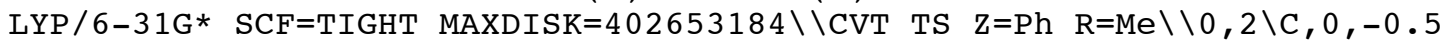
$7808,-0.143148,-0.244012 \backslash \mathrm{S}, 0,-1.505603,1.119567,-0.866067 \backslash \mathrm{S}, 0,-1.30581$ $6,-1.626119,0.406607 \backslash \mathrm{C}, 0,-3.040697,-1.540575,-0.137481 \backslash \mathrm{H}, 0,-3.500437,-$ $2.470583,0.208563 \backslash \mathrm{H}, 0,-3.101481,-1.476508,-1.225897 \backslash \mathrm{H}, 0,-3.551514,-0.6$ $84353,0.305426 \backslash \mathrm{C}, 0,0.889354,-0.08226,-0.122188 \backslash \mathrm{C}, 0,1.678084,-1.249355$, $-0.016061 \backslash \mathrm{C}, 0,3.062635,-1.169962,0.105786 \backslash \mathrm{C}, 0,3.700608,0.071678,0.1254$ $12 \backslash \mathrm{C}, 0,2.936252,1.236884,0.014862 \backslash \mathrm{C}, 0,1.553678,1.163534,-0.109968 \backslash \mathrm{H}, 0$, $0.965122,2.070015,-0.197638 \backslash \mathrm{H}, 0,3.421374,2.209337,0.028266 \backslash \mathrm{H}, 0,4.78138$ $9,0.131497,0.220309 \backslash \mathrm{H}, 0,3.645926,-2.084185,0.175862 \backslash \mathrm{H}, 0,1.205573,-2.22$ $5081,-0.058678 \backslash \mathrm{C}, 0,-2.213909,2.510263,1.08495 \backslash \mathrm{H}, 0,-2.564493,1.742852,1$ $.76601 \backslash \mathrm{H}, 0,-2.968211,3.111466,0.588552 \backslash \mathrm{H}, 0,-1.278078,2.998013,1.332776$ $\backslash \backslash$ Version $=x 86-$ Linux-G03RevB .03 $\backslash$ State $=2-A \backslash H F=-1145.8865856 \backslash S 2=0.795775 \backslash$ $\mathrm{S} 2-1=0 . \backslash \mathrm{S} 2 \mathrm{~A}=0.750657 \backslash \mathrm{RMSD}=8.304 \mathrm{e}-09 \backslash \mathrm{Dipole}=-0.0780146,-0.0713889,0.405$ $6126 \backslash P G=C 01 \quad[X(C 9 H 11 S 2)] \backslash \backslash @$

\section{Formal TS: $\cdot \mathrm{CH}_{3}+\mathrm{S}=\mathrm{C}\left(\mathrm{CH}_{2} \mathrm{Ph}\right) \mathrm{SCH}_{3}$ (3)}

$1 \backslash 1 \backslash G I N C-S C 35 \backslash F T S \backslash U B 3 L Y P \backslash 6-31 G(d) \backslash C 10 H 13 S 2(2) \backslash M L C 501 \backslash 02-A u g-2002 \backslash 0 \backslash \backslash \# N$ B3LYP $/ 6-31 \mathrm{G} * \mathrm{SCF}=(\mathrm{TIGHT}, \mathrm{MAXCYC}=100, \mathrm{QC}) \quad \mathrm{MAXDISK}=131072000$ OPT $=($ NOEIGEN TEST, TS , CALCFC, MAXCYC=100) FREQ GEOM=CHECK GUESS=READ \\TS: ch3+RAFT Ag ent $\mathrm{S}=\mathrm{C}(\mathrm{MePH})-\mathrm{SMe}(\mathrm{Z}=\mathrm{MePh}, \mathrm{R}=\mathrm{Me}) \mathrm{C} 1 \mathrm{a} \backslash \backslash 0,2 \backslash \mathrm{C},-0.6734324477,-1.2762810842$ $, 0.0593583044 \backslash \mathrm{S},-0.7151039105,-1.348902003,1.7067524775 \backslash \mathrm{C}, 0.6282146512$ $,-1.2943869344,-0.742584905 \backslash \mathrm{H}, 0.4295984965,-1.6411072478,-1.764409549 \backslash$ $\mathrm{H}, 1.2947929131,-2.0228502188,-0.2714782264 \backslash \mathrm{C}, 1.3044597475,0.068069859$, $-0.790575973 \backslash \mathrm{C}, 1.000059286,0.9827830203,-1.8062631443 \backslash \mathrm{C}, 1.6098161522,2$ $.2376503493,-1.8413442779 \backslash \mathrm{C}, 2.5340092194,2.5954129374,-0.8583820104 \backslash \mathrm{C}$, $2.8458307132,1.6895172666,0.1574672836 \backslash \mathrm{C}, 2.2351155319,0.4347070359,0.1$ $903054016 \backslash \mathrm{H}, 2.479397928,-0.2678202918,0.9825637318 \backslash \mathrm{H}, 3.5701903915,1.95$ $64357365,0.9226720875 \backslash \mathrm{H}, 3.0117678805,3.5710849207,-0.88646757 \backslash \mathrm{H}, 1.3646$ $059467,2.9338564362,-2.6391571605 \backslash \mathrm{H}, 0.2817023158,0.7107386342,-2.57603$ $72346 \backslash \mathrm{S},-2.071778809,-1.098339179,-0.9966650616 \backslash \mathrm{C},-3.4787007079,-1.026$ $3328784,0.1534128253 \backslash \mathrm{H},-4.3749889159,-0.9401614384,-0.4664470118 \backslash \mathrm{H},-3$. $3902883584,-0.1597887899,0.8115491662 \backslash \mathrm{H},-3.5222653739,-1.9356449714,0$. $7564967295 \backslash \mathrm{C},-0.5130190395,1.1365830697,2.9441321055 \backslash \mathrm{H},-0.2597252855,0$ $.6954423281,3.900520249 \backslash \mathrm{H}, 0.2794669366,1.4963963393,2.300690745 \backslash \mathrm{H},-1.5$ $282500012,1.4729416278,2.7749517293 \backslash \backslash V e r s i o n=D E C-A X P-O S F / 1-G 98 R e v A .11$. $3 \backslash \mathrm{HF}=-1185.1928377 \backslash \mathrm{S} 2=0.771496 \backslash \mathrm{S} 2-1=0 . \backslash \mathrm{S} 2 \mathrm{~A}=0.750137 \backslash \mathrm{RMSD}=0.000 \mathrm{e}+00 \backslash \mathrm{RMS}$ $\mathrm{F}=4.009 e-06 \backslash \mathrm{Dipole}=-0.2475123,0.3693606,-0.3204086 \backslash \mathrm{PG}=\mathrm{C} 01 \quad[\mathrm{X}(\mathrm{C} 10 \mathrm{H} 13 \mathrm{~S} 2)$ ]$\backslash \backslash @$

\section{Variational TS: $\cdot \mathrm{CH}_{3}+\mathrm{S}=\mathrm{C}\left(\mathrm{CH}_{2} \mathrm{Ph}\right) \mathrm{SCH}_{3}(3)$}

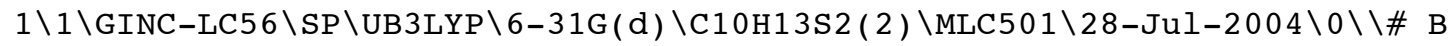

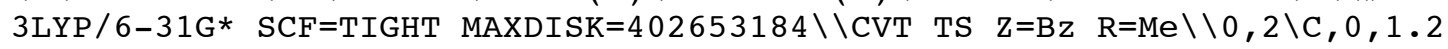
$40858,-0.160902,-0.683826 \backslash \mathrm{S}, 0,1.648135,1.457075,-0.582075 \backslash \mathrm{C}, 0,0.028196$ $,-0.660486,-1.464504 \backslash \mathrm{H}, 0,0.163,-1.71968,-1.717942 \backslash \mathrm{H}, 0,-0.016812,-0.102$ $779,-2.405505 \backslash \mathrm{C}, 0,-1.273091,-0.481,-0.69809 \backslash \mathrm{C}, 0,-1.736938,-1.474957,0$. 
$172717 \backslash \mathrm{C}, 0,-2.918525,-1.295837,0.893344 \backslash \mathrm{C}, 0,-3.652931,-0.116836,0.7540$ $24 \backslash \mathrm{C}, 0,-3.19996,0.879329,-0.113264 \backslash \mathrm{C}, 0,-2.018941,0.696939,-0.834448 \backslash \mathrm{H}$, $0,-1.669214,1.473273,-1.509915 \backslash \mathrm{H}, 0,-3.769852,1.796972,-0.234972 \backslash \mathrm{H}, 0,-4$ $.574608,0.022325,1.312746 \backslash \mathrm{H}, 0,-3.266581,-2.07941,1.561423 \backslash \mathrm{H}, 0,-1.16969$ $7,-2.395728,0.286887 \backslash \mathrm{S}, 0,2.063967,-1.481336,0.143472 \backslash \mathrm{C}, 0,3.420186,-0.6$ $64486,1.039089 \backslash \mathrm{H}, 0,3.971125,-1.459759,1.548115 \backslash \mathrm{H}, 0,3.027145,0.044728,1$ $.769928 \backslash \mathrm{H}, 0,4.07639,-0.140194,0.341429 \backslash \mathrm{C}, 0,0.528178,2.699803,1.252575 \backslash$ $\mathrm{H}, 0,0.73424,3.696541,0.874955 \backslash \mathrm{H}, 0,-0.489508,2.338084,1.158676 \backslash \mathrm{H}, 0,1.08$ $8556,2.384395,2.126122 \backslash \backslash$ Version=x86-Linux-G03RevB .03 $\backslash$ State $=2-A \backslash H F=-118$ $5.1951373 \backslash \mathrm{S} 2=0.788418 \backslash \mathrm{S} 2-1=0 . \backslash \mathrm{S} 2 \mathrm{~A}=0.75023 \backslash \mathrm{RMSD}=6.369 \mathrm{e}-09 \backslash \mathrm{Dipole}=-0.080$ $3874,-0.1328092,0.5322179 \backslash \mathrm{PG}=\mathrm{C} 01 \quad[\mathrm{X}(\mathrm{C} 10 \mathrm{H} 13 \mathrm{~S} 2)] \backslash \backslash @$

\section{Formal TS: $\cdot \mathrm{CH}_{2} \mathrm{COOCH}_{3}+\mathrm{S}=\mathrm{C}\left(\mathrm{CH}_{3}\right) \mathrm{SCH}_{3}(4)$}

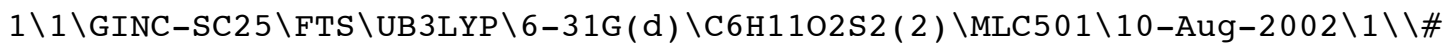
UB3LYP /6-31G* FOPT=(TS, CALCFC , NOEIGENTEST, Z-MATRIX, MAXCYC $=100)$ MAXDIS $\mathrm{K}=131072000 \mathrm{FREQ} \quad \mathrm{SCF}=(\mathrm{QC}, \mathrm{TIGHT}, \mathrm{MAXCYC}=100) \backslash \backslash \mathrm{MA}+\mathrm{S}=\mathrm{C}(\mathrm{CH} 3)-\mathrm{SCH} 3 \mathrm{TS} \mathrm{C} 1 \mathrm{C} / / \mathrm{B}$ $3 \backslash \backslash 0,2 \backslash S \backslash C, 1, B 1 \backslash \mathrm{C}, 2$, $\mathrm{B} 2,1, \mathrm{~A} 1 \backslash \mathrm{S}, 2, \mathrm{~B} 3,1, \mathrm{~A} 2,3, \mathrm{D} 1,0 \backslash \mathrm{C}, 4, \mathrm{~B} 4,2, \mathrm{~A} 3,1, \mathrm{D} 2,0 \backslash \mathrm{H}, 5$, $\mathrm{B} 5,4, \mathrm{~A} 4,2, \mathrm{D} 3,0 \backslash \mathrm{H}, 5, \mathrm{~B} 6,4, \mathrm{~A} 5,6, \mathrm{D} 4,0 \backslash \mathrm{H}, 5, \mathrm{~B} 7,4, \mathrm{~A} 6,6, \mathrm{D} 5,0 \backslash \mathrm{H}, 3, \mathrm{~B} 8,2, \mathrm{~A} 7,1, \mathrm{D} 6$, $0 \backslash \mathrm{H}, 3, \mathrm{~B} 9,2, \mathrm{~A} 8,9, \mathrm{D} 7,0 \backslash \mathrm{H}, 3, \mathrm{~B} 10,2, \mathrm{~A} 9,9, \mathrm{D} 8,0 \backslash \mathrm{C}, 1, \mathrm{~B} 11,2, \mathrm{~A} 10,3, \mathrm{D} 9,0 \backslash \mathrm{C}, 12, \mathrm{~B} 12$ $, 1, \mathrm{~A} 11,2, \mathrm{D} 10,0 \backslash \mathrm{H}, 12, \mathrm{~B} 13,1, \mathrm{~A} 12,2, \mathrm{D} 11,0 \backslash \mathrm{H}, 12, \mathrm{~B} 14,1, \mathrm{~A} 13,2, \mathrm{D} 12,0 \backslash 0,13, \mathrm{~B} 15$, $12, \mathrm{~A} 14,1, \mathrm{D} 13,0 \backslash \mathrm{O}, 13, \mathrm{~B} 16,12, \mathrm{~A} 15,16, \mathrm{D} 14,0 \backslash \mathrm{C}, 16, \mathrm{~B} 17,13, \mathrm{~A} 16,12, \mathrm{D} 15,0 \backslash \mathrm{H}, 18$, $\mathrm{B} 18,16, \mathrm{~A} 17,13, \mathrm{D} 16,0 \backslash \mathrm{H}, 18, \mathrm{~B} 19,16, \mathrm{~A} 18,19, \mathrm{D} 17,0 \backslash \mathrm{H}, 18, \mathrm{~B} 20,16, \mathrm{~A} 19,19, \mathrm{D} 18,0 \backslash$ $\backslash \mathrm{D} 12=-146.32032858 \backslash \mathrm{D} 11=94.08987388 \backslash \mathrm{D} 10=-27.85838677 \backslash \mathrm{B} 1=1.66461947 \backslash \mathrm{B} 2=1$ $.51126012 \backslash \mathrm{B} 3=1.74900026 \backslash \mathrm{B} 4=1.82095835 \backslash \mathrm{B} 5=1.09227776 \backslash \mathrm{B} 6=1.09167982 \backslash \mathrm{B} 7=1$ $.0932166 \backslash \mathrm{B} 8=1.09568854 \backslash \mathrm{B} 9=1.09767465 \backslash \mathrm{B} 10=1.0932799 \backslash \mathrm{B} 11=2.54739979 \backslash \mathrm{B} 12=$ $1.45220452 \backslash \mathrm{B} 13=1.08230872 \backslash \mathrm{B} 14=1.08230894 \backslash \mathrm{B} 15=1.36360692 \backslash \mathrm{B} 16=1.22389523$ $\backslash \mathrm{B} 17=1.43443661 \backslash \mathrm{B} 18=1.09350179 \backslash \mathrm{B} 19=1.09085703 \backslash \mathrm{B} 20=1.09387802 \backslash \mathrm{A} 1=122.18$ $861295 \backslash \mathrm{A} 2=125.28066117 \backslash \mathrm{A} 3=104.649247 \backslash \mathrm{A} 4=109.91565775 \backslash \mathrm{A} 5=110.23864979 \backslash \mathrm{A}$ $6=105.7280661 \backslash A 7=112.60553443 \backslash A 8=108.57794282 \backslash A 9=110.17273798 \backslash A 10=106$. $67116168 \backslash \mathrm{A} 11=102.33779215 \backslash \mathrm{A} 12=95.24428173 \backslash \mathrm{A} 13=93.22460387 \backslash \mathrm{A} 14=111.7747$ $5861 \backslash A 15=125.5060675 \backslash A 16=115.00649094 \backslash A 17=110.71840018 \backslash A 18=105.5701492$ $\backslash A 19=110.68880698 \backslash D 1=175.46188505 \backslash D 2=-1.78685275 \backslash D 3=-65.27251438 \backslash D 4=12$ $1.94047782 \backslash D 5=-118.99446038 \backslash D 6=-163.86266204 \backslash D 7=-119.85935356 \backslash D 8=122.2$ $9441828 \backslash \mathrm{D} 9=-86.41821514 \backslash \mathrm{D} 13=89.26328774 \backslash \mathrm{D} 14=-178.35964098 \backslash \mathrm{D} 15=-179.626$ $48464 \backslash \mathrm{D} 16=-58.29594673 \backslash \mathrm{D} 17=-119.57390663 \backslash \mathrm{D} 18=120.8420261 \backslash \backslash$ Version=DEC-

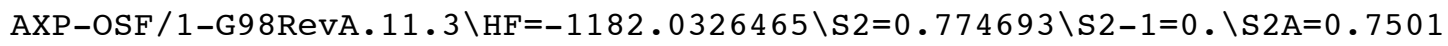
$99 \backslash \mathrm{RMSD}=0.000 \mathrm{e}+00 \backslash \mathrm{RMSF}=6.575 \mathrm{e}-05 \backslash \mathrm{Dipole}=0.3064759,0.0137703,0.6512708 \backslash$ $\mathrm{PG}=\mathrm{C} 01[\mathrm{X}(\mathrm{C} 6 \mathrm{H} 1102 \mathrm{~S} 2)] \backslash \backslash @$

\section{Variational TS: $\bullet \mathrm{CH}_{2} \mathrm{COOCH}_{3}+\mathrm{S}=\mathrm{C}\left(\mathrm{CH}_{3}\right) \mathrm{SCH}_{3}(4)$}

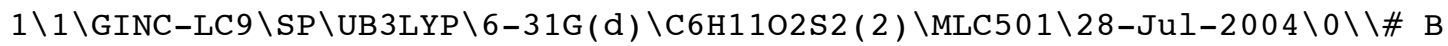

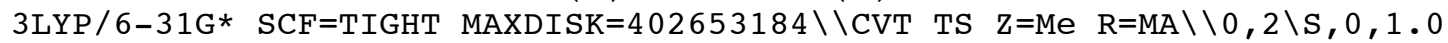
$24863,1.61094,-0.462361 \backslash \mathrm{C}, 0,1.384357,0.369403,0.599279 \backslash \mathrm{C}, 0,1.39415,0.5$ $71669,2.096167 \backslash \mathrm{S}, 0,1.668014,-1.295244,0.143432 \backslash \mathrm{C}, 0,1.450708,-1.324328$, $-1.664476 \backslash \mathrm{H}, 0,0.414014,-1.094325,-1.92009 \backslash \mathrm{H}, 0,2.12724,-0.6105,-2.13824$ $7 \backslash \mathrm{H}, 0,1.699689,-2.342203,-1.976051 \backslash \mathrm{H}, 0,1.902687,-0.241564,2.625981 \backslash \mathrm{H}, 0$ $, 0.357617,0.619594,2.454932 \backslash \mathrm{H}, 0,1.877418,1.52027,2.346257 \backslash \mathrm{C}, 0,-1.40902$ $7,1.66569,-0.728477 \backslash \mathrm{C}, 0,-1.829952,0.289664,-0.489987 \backslash \mathrm{H}, 0,-1.614289,2.4$ $10797,0.030236 \backslash \mathrm{H}, 0,-1.386552,1.983246,-1.763788 \backslash 0,0,-2.0845,0.062735,0$ $.829095 \backslash 0,0,-1.912322,-0.58609,-1.339479 \backslash \mathrm{C}, 0,-2.460927,-1.28371,1.1524$ $95 \backslash \mathrm{H}, 0,-3.35715,-1.578464,0.599884 \backslash \mathrm{H}, 0,-2.656814,-1.283047,2.225563 \backslash \mathrm{H}$, $0,-1.651176,-1.978428,0.911258 \backslash \backslash$ Version=x86-Linux-G03RevB.03 $\backslash$ State $=2-A$ $\backslash \mathrm{HF}=-1182.0329621 \backslash \mathrm{S} 2=0.776838 \backslash \mathrm{S} 2-1=0 . \backslash \mathrm{S} 2 \mathrm{~A}=0.750194 \backslash \mathrm{RMSD}=6.975 \mathrm{e}-09 \backslash \mathrm{Dipo}$ $l e=0.0601861,-0.2747133,0.6295681 \backslash \mathrm{PG}=\mathrm{C} 01 \quad[\mathrm{X}(\mathrm{C} 6 \mathrm{H} 1102 \mathrm{~S} 2)] \backslash \backslash @$

\section{Formal and Variational TS: $\cdot \mathrm{CH}_{2} \mathrm{Ph}+\mathrm{S}=\mathrm{C}\left(\mathrm{CH}_{3}\right) \mathrm{SCH}_{3}(5)$}

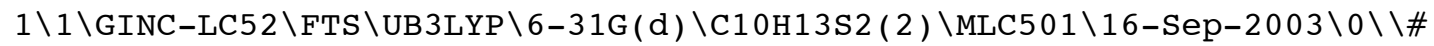
B3LYP /6-31G* OPT $=($ TS , CALCFC , NOEIGENTEST , MAXCYC $=200)$ FREQ MAXDISK=13421 


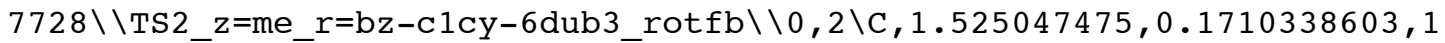
$.59539186 \overline{2} 5 \backslash \mathrm{C}, \overline{3} .0118901942,0.3 \overline{5} 35395392,1.3904232235 \backslash \mathrm{S}, 0.6872448671,1$. $7115574836,1.752878681 \backslash \mathrm{C},-1.0682319704,1.2563830531,1.9001211692 \backslash \mathrm{S}, 0.7$ $902191136,-1.3264299546,1.5503119245 \backslash \mathrm{C}, 0.421695787,-2.0235553781,-0.75$ $47879988 \backslash \mathrm{H}, 0.0051714417,-2.9675008301,-0.4155837462 \backslash \mathrm{H}, 1.4722214036,-2$. $0522067908,-1.0240516122 \backslash \mathrm{C},-0.4392677923,-1.0865504473,-1.4214981173 \backslash \mathrm{C}$ $, 0.0939053795,-0.0039954732,-2.1621144444 \backslash \mathrm{C},-1.8491494017,-1.200579381$ $1,-1.3428622901 \backslash \mathrm{C},-0.7395093626,0.9102763696,-2.7965892312 \backslash \mathrm{C},-2.677797$ $5349,-0.2845250225,-1.9795557337 \backslash \mathrm{C},-2.1294283348,0.7769171445,-2.71040$ $65416 \backslash \mathrm{H}, 1.1734428168,0.1035396247,-2.2356675008 \backslash \mathrm{H},-2.2799143999,-2.022$ $282478,-0.7758268226 \backslash \mathrm{H},-0.3068838654,1.7307872499,-3.3630872344 \backslash \mathrm{H},-1.2$ $219069044,0.6080458239,2.7652799133 \backslash \mathrm{H},-1.4127365254,0.7504332871,0.996$ $1592209 \backslash \mathrm{H},-2.779329731,1.4910885218,-3.2083176033 \backslash \mathrm{H},-3.7569553808,-0.3$ $960952829,-1.9117897589 \backslash \mathrm{H}, 3.556655899,-0.4905459968,1.8214966065 \backslash \mathrm{H}, 3.3$ $804382922,1.283235975,1.8396443268 \backslash \mathrm{H}, 3.2464627274,0.3899271391,0.31707$ $37416 \backslash \mathrm{H},-1.6110160994,2.1958677052,2.0348893917 \backslash \backslash$ Version=x86-Linux-G03 RevB.03 \State $=2-A \backslash H F=-1185.2157971 \backslash \mathrm{S} 2=0.787974 \backslash \mathrm{S} 2-1=0 . \backslash \mathrm{S} 2 \mathrm{~A}=0.750896 \backslash \mathrm{RM}$ $\mathrm{SD}=6.237 \mathrm{e}-09 \backslash \mathrm{RMSF}=2.222 \mathrm{e}-06 \backslash \mathrm{Dipole}=-0.2394189,0.3211102,-0.4721292 \backslash \mathrm{PG}=$ $\mathrm{C} 01[\mathrm{X}(\mathrm{C} 10 \mathrm{H} 13 \mathrm{~S} 2)] \backslash \backslash @$

\section{Formal TS: $\cdot C\left(\mathrm{CH}_{3}\right)_{2} \mathrm{CN}+\mathrm{S}=\mathrm{C}\left(\mathrm{CH}_{3}\right) \mathrm{SCH}_{3}(6)$}

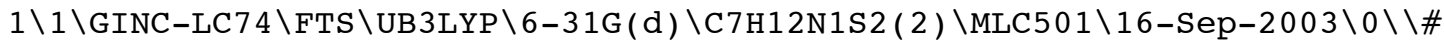
B3LYP /6-31G* OPT $=(T S, C A L C F C, N O E I G E N T E S T, M A X C Y C=200)$ FREQ MAXDISK=1342 $17728 \backslash \backslash \mathrm{TS} 2 \_z=m e \_r=m c n-c 1 a-6$ dub3_rotfb $\backslash \backslash 0,2 \backslash \mathrm{C},-1.7287615609,-0.86068378$ $22,0.43778 \overline{7} 2905 \backslash \mathrm{S}, 0.627593931,-\overline{0} .9365436677,0.5933962363 \backslash \mathrm{C}, 1.308638446$ $7,0.5980755948,0.5749746494 \backslash \mathrm{S}, 1.8578853213,1.4481756674,-0.8537208339 \backslash$ $\mathrm{C}, 1.5342131427,0.3123035094,-2.2402112739 \backslash \mathrm{C}, 1.4552086511,1.434077253,1$ $.8273578953 \backslash \mathrm{C},-1.9274286624,-0.3219991715,-0.8688453023 \backslash \mathrm{C},-2.167590627$ $8,0.027587859,1.5820705531 \backslash \mathrm{C},-2.0013540909,-2.3438196216,0.5807859676 \backslash$ $\mathrm{N},-2.0568601892,0.1261021593,-1.9398990919 \backslash \mathrm{H},-1.8305518152,1.058766847$ $2,1.4458513862 \backslash \mathrm{H},-1.5525718306,-2.9172740846,-0.2348755514 \backslash \mathrm{H},-3.265770$ $2733,0.0420131753,1.6519486029 \backslash \mathrm{H},-1.7714128261,-0.3526747361,2.5280906$ $086 \backslash \mathrm{H},-1.6028339853,-2.7110736862,1.5319374627 \backslash \mathrm{H},-3.0845330014,-2.5330$ $982052,0.5724408024 \backslash \mathrm{H}, 1.9786374922,0.7881483779,-3.1183573053 \backslash \mathrm{H}, 0.4604$ $689884,0.1832627533,-2.3889504801 \backslash \mathrm{H}, 2.0110263511,-0.6527100328,-2.0574$ $399666 \backslash \mathrm{H}, 1.5521170763,0.7866947354,2.7023957255 \backslash \mathrm{H}, 2.3273338477,2.09625$ $84604,1.7750845892 \backslash \mathrm{H}, 0.5708914735,2.0696094401,1.9728426512 \backslash \backslash$ Version $=\mathrm{x}$ 86-Linux-G03RevB.03 State $=2-\mathrm{A} \backslash \mathrm{HF}=-1125.0357271 \backslash \mathrm{S} 2=0.778039 \backslash \mathrm{S} 2-1=0 . \backslash \mathrm{S} 2 \mathrm{~A}$ $=0.750271 \backslash \mathrm{RMSD}=7.088 \mathrm{e}-09 \backslash \mathrm{RMSF}=8.624 \mathrm{e}-06 \backslash \mathrm{Dipole}=0.1092093,-0.1567562,1$. $0059605 \backslash \mathrm{PG}=\mathrm{C} 01 \quad[\mathrm{X}(\mathrm{C} 7 \mathrm{H} 12 \mathrm{~N} 1 \mathrm{~S} 2)] \backslash \backslash \mathrm{Q}$

\section{Variational TS: $\cdot \mathrm{C}\left(\mathrm{CH}_{3}\right)_{2} \mathrm{CN}+\mathrm{S}=\mathrm{C}\left(\mathrm{CH}_{3}\right) \mathrm{SCH}_{3}(6)$}

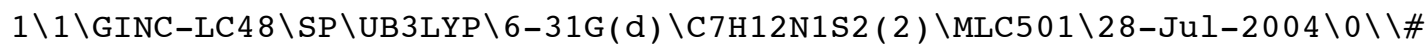

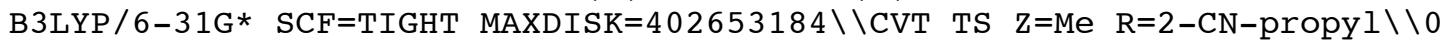
, $2 \backslash \mathrm{S}, 0,0.028824,-0.634127,1.118379 \backslash \mathrm{C}, 0,-1.286108,-0.866676,0.114097 \backslash \mathrm{C}$, $0,-1.553879,-2.184528,-0.580364 \backslash \mathrm{S}, 0,-2.461137,0.355598,-0.321779 \backslash \mathrm{C}, 0,-$ $1.9224,1.863714,0.54553 \backslash \mathrm{H}, 0,-1.079503,-2.1976,-1.57096 \backslash \mathrm{H}, 0,-1.137361,-$ $3.008195,0.004269 \backslash \mathrm{H}, 0,-2.626068,-2.356953,-0.729592 \backslash \mathrm{H}, 0,-0.9791,2.2271$ $38,0.13332 \backslash \mathrm{H}, 0,-2.713607,2.598485,0.374617 \backslash \mathrm{H}, 0,-1.81897,1.667333,1.614$ $552 \backslash \mathrm{C}, 0,2.014314,-0.008781,-0.134818 \backslash \mathrm{C}, 0,1.621849,1.25561,-0.651795 \backslash \mathrm{C}$, $0,2.164367,-1.125236,-1.14099 \backslash \mathrm{C}, 0,2.970424,0.012725,1.03552 \backslash \mathrm{H}, 0,1.3428$ $97,-1.133443,-1.862545 \backslash \mathrm{H}, 0,3.101753,-1.004474,-1.705511 \backslash \mathrm{H}, 0,2.198169,-$ $2.092531,-0.63127 \backslash \mathrm{H}, 0,3.989404,0.241197,0.689512 \backslash \mathrm{H}, 0,2.994325,-0.96670$ $4,1.523947 \backslash \mathrm{H}, 0,2.688964,0.768672,1.773688 \backslash \mathrm{N}, 0,1.272101,2.290369,-1.068$ 96\\Version $=x 86-$ Linux $-G 03 R e v B .03 \backslash$ State $=2-A \backslash H F=-1125.0359408 \backslash S 2=0.77909$ $8 \backslash \mathrm{S} 2-1=0 . \backslash \mathrm{S} 2 \mathrm{~A}=0.75033 \backslash \mathrm{RMSD}=6.354 \mathrm{e}-09 \backslash \mathrm{Dipole}=0.1730284,-1.0040893,0.143$ $0859 \backslash \mathrm{PG}=\mathrm{C} 01 \quad[\mathrm{X}(\mathrm{C} 7 \mathrm{H} 12 \mathrm{~N} 1 \mathrm{~S} 2)] \backslash \backslash \mathrm{a}$ 
Table S2. Full Rotational Potentials $\left(\mathrm{kJ} \mathrm{mol}^{-1}\right)$ for those Modes Treated As Hindered

Internal Rotations ${ }^{a}$

\begin{tabular}{|c|c|c|c|c|c|c|c|c|c|c|c|c|c|}
\hline$\theta(\mathrm{rad})$ & $1 \mathrm{~b}$ & $2 b$ & $2 c$ & $3 b$ & $3 c$ & $3 d$ & $4 b$ & $4 d$ & $4 e$ & $5 b$ & $5 \mathrm{~d}$ & $6 \mathrm{~b}$ & $6 \mathrm{~d}$ \\
\hline 0.00 & 0.0 & 0.0 & 0.0 & 0.0 & 0.0 & 0.0 & 0.0 & 0.0 & 0.0 & 0.0 & 0.0 & 0.0 & 0.0 \\
\hline 0.17 & 1.3 & 0.9 & 0.6 & 1.2 & 0.1 & -0.1 & 1.3 & 0.6 & 1.9 & 1.2 & 0.2 & 1.1 & 0.4 \\
\hline 0.35 & 4.3 & 3.3 & 2.5 & 4.1 & 1.2 & 0.5 & 5.5 & 1.4 & 7.3 & 4.7 & 0.8 & 4.3 & 1.4 \\
\hline 0.52 & 8.6 & 7.1 & 5.6 & 8.3 & 3.1 & 1.6 & 11.7 & 2.6 & 15.4 & 9.4 & 1.4 & 9.3 & 2.8 \\
\hline 0.70 & 13.7 & 11.9 & 9.8 & 13.5 & 5.1 & 3.2 & 19.3 & 3.8 & 24.9 & 13.2 & 2.3 & 15.6 & 4.8 \\
\hline 0.87 & 19.5 & 17.2 & 14.0 & 19.5 & 6.5 & 4.7 & 28.7 & 5.0 & 33.4 & 19.1 & 3.1 & 22.8 & 6.8 \\
\hline 1.05 & 25.7 & 22.7 & 17.0 & 26.0 & 7.7 & 6.2 & 38.4 & 6.7 & 41.4 & 25.4 & 3.3 & 30.1 & 8.4 \\
\hline 1.22 & 31.5 & 27.2 & 17.4 & 32.4 & 8.6 & 7.5 & 46.1 & 9.5 & 48.4 & 31.5 & 3.1 & 36.9 & 9.3 \\
\hline 1.40 & 36.2 & 29.9 & 15.4 & 37.9 & 8.9 & 8.5 & 52.4 & 5.8 & 53.5 & 36.2 & 2.6 & 42.2 & 9.6 \\
\hline 1.57 & 39.1 & 30.4 & 12.0 & 42.1 & 8.8 & 9.3 & 55.7 & 4.8 & 56.1 & 38.8 & 1.8 & 50.9 & 9.5 \\
\hline 1.75 & 39.9 & 23.5 & 8.2 & 44.6 & 8.3 & 9.3 & 55.9 & 4.1 & 56.1 & 39.1 & 1.7 & 45.7 & 9.6 \\
\hline 1.92 & 38.9 & 20.3 & 4.9 & 45.9 & 7.3 & 8.9 & 47.0 & 3.8 & 53.9 & 37.4 & 1.7 & 38.5 & 9.9 \\
\hline 2.09 & 36.1 & 18.0 & 2.6 & 45.5 & 6.4 & 8.1 & 42.5 & 4.2 & 50.6 & 33.0 & 2.1 & 30.3 & 10.1 \\
\hline 2.27 & 26.8 & 16.8 & 1.3 & 32.3 & 5.3 & 7.1 & 37.7 & 5.8 & 47.0 & 28.4 & 2.6 & 22.2 & 10.5 \\
\hline 2.44 & 21.6 & 16.7 & 1.1 & 27.2 & 4.3 & 6.1 & 33.0 & 8.1 & 42.6 & 23.6 & 3.2 & 16.0 & 11.1 \\
\hline 2.62 & 17.1 & 17.9 & 1.2 & 22.6 & 3.1 & 4.4 & 28.5 & 10.2 & 38.0 & 19.2 & 3.8 & 12.4 & 11.6 \\
\hline 2.79 & 13.7 & 20.4 & 1.0 & 19.4 & 2.5 & 2.4 & 25.0 & 11.3 & 34.4 & 15.8 & 4.2 & 11.0 & 11.8 \\
\hline 2.97 & 11.7 & 23.8 & 0.3 & 17.4 & 2.5 & 0.8 & 22.7 & 12.1 & 32.0 & 13.7 & 4.3 & 11.0 & 11.9 \\
\hline 3.14 & 11.1 & 27.8 & 0.0 & 16.4 & 2.9 & 0.0 & 21.9 & 12.8 & 31.0 & 13.0 & 4.2 & 11.7 & 11.5 \\
\hline 3.32 & 11.5 & 15.6 & 0.6 & 16.7 & 3.5 & 0.0 & 22.8 & 13.3 & 31.3 & 13.4 & 4.1 & 13.5 & 10.8 \\
\hline 3.49 & 13.3 & 15.1 & 2.5 & 18.7 & 4.0 & 0.5 & 25.1 & 13.5 & 32.9 & 15.1 & 3.7 & 16.6 & 9.5 \\
\hline 3.67 & 16.5 & 16.2 & 5.6 & 20.3 & 4.3 & 1.6 & 28.8 & 13.2 & 35.5 & 18.2 & 3.3 & 21.0 & 8.0 \\
\hline 3.84 & 20.6 & 18.9 & 9.8 & 23.8 & 4.6 & 3.0 & 33.1 & 12.6 & 39.1 & 22.7 & 2.7 & 26.1 & 6.2 \\
\hline 4.01 & 25.3 & 23.0 & 14.0 & 28.1 & 5.0 & 4.4 & 38.4 & 11.0 & 43.0 & 27.9 & 2.3 & 33.4 & 4.9 \\
\hline 4.19 & 29.8 & 27.8 & 17.0 & 32.2 & 5.6 & 5.9 & 44.5 & 8.8 & 46.3 & 33.4 & 2.0 & 38.8 & 4.1 \\
\hline 4.36 & 33.6 & 32.7 & 17.4 & 35.7 & 6.5 & 7.3 & 49.9 & 7.1 & 48.6 & 38.2 & 2.0 & 43.4 & 3.7 \\
\hline 4.54 & 36.8 & 29.5 & 15.4 & 37.8 & 7.2 & 8.5 & 54.0 & 6.3 & 50.0 & 0.0 & 2.1 & 53.5 & 3.6 \\
\hline 4.71 & 38.6 & 26.3 & 12.0 & 37.8 & 7.3 & 9.3 & 56.6 & 6.5 & 49.9 & 40.0 & 2.3 & 52.7 & 3.8 \\
\hline 4.89 & 38.0 & 22.3 & 8.2 & 35.3 & 7.0 & 9.3 & 50.7 & 7.4 & 47.5 & 37.2 & 2.3 & 48.7 & 4.0 \\
\hline 5.06 & 30.4 & 17.9 & 4.9 & 30.6 & 6.4 & 8.7 & 46.5 & 8.5 & 42.8 & 32.4 & 2.0 & 42.0 & 4.1 \\
\hline 5.24 & 24.5 & 13.7 & 2.6 & 24.9 & 5.8 & 7.8 & 40.8 & 9.1 & 36.0 & 26.5 & 1.5 & 33.4 & 4.1 \\
\hline 5.41 & 18.2 & 9.7 & 1.3 & 19.0 & 5.3 & 6.8 & 33.0 & 8.3 & 28.1 & 20.2 & 1.1 & 24.3 & 3.9 \\
\hline 5.59 & 12.4 & 6.0 & 1.1 & 13.5 & 4.8 & 5.9 & 20.0 & 6.2 & 19.9 & 14.0 & 0.7 & 15.8 & 3.1 \\
\hline 5.76 & 7.4 & 3.1 & 1.2 & 8.0 & 3.7 & 4.2 & 11.8 & 3.5 & 11.9 & 8.6 & 0.4 & 8.9 & 1.8 \\
\hline 5.93 & 3.5 & 1.4 & 1.0 & 3.6 & 2.1 & 2.4 & 5.4 & 0.0 & 5.4 & 4.1 & 0.2 & 3.9 & 0.8 \\
\hline 6.11 & 0.8 & 1.1 & 0.3 & 0.8 & 0.7 & 0.8 & 1.4 & 0.0 & 1.2 & 1.1 & 0.2 & 1.2 & 0.1 \\
\hline 6.28 & 0.0 & 0.0 & 0.0 & 0.0 & 0.0 & 0.0 & 0.0 & 0.0 & 0.0 & 0.0 & 0.0 & 0.0 & 0.0 \\
\hline
\end{tabular}

${ }^{a}$ Potentials Calculated at the B3-LYP/6-31G(d) level of theory as relaxed scans for the rotations depicted in Figures S1-3. 
Table S2 continued

\begin{tabular}{|c|c|c|c|c|c|c|c|c|c|c|c|c|}
\hline$\theta(\mathrm{rad})$ & $7 \mathrm{~b}$ & $7 \mathrm{~d}$ & $8 \mathrm{~b}$ & $8 c$ & $9 \mathrm{~b}$ & $9 c$ & $9 d$ & $9 \mathrm{e}$ & $10 \mathrm{~b}$ & $10 d^{b}$ & $10 e^{b}$ & $10 f$ \\
\hline 0.00 & 0.0 & 0.0 & 0.0 & 0.0 & 0.0 & 0.0 & 0.0 & 0.0 & 0.0 & 0.0 & 0.0 & 0.0 \\
\hline 0.17 & 0.3 & 0.3 & 0.4 & 0.9 & 0.2 & 0.5 & 0.2 & 0.2 & 0.4 & 0.5 & 0.5 & 0.5 \\
\hline 0.35 & 1.5 & 1.5 & 1.4 & 3.2 & 0.6 & 2.0 & 0.8 & 0.5 & 1.3 & 1.6 & 1.8 & 2.2 \\
\hline 0.52 & 3.1 & 3.1 & 3.4 & 6.5 & 1.2 & 4.1 & 1.5 & 0.8 & 2.4 & 2.8 & 3.4 & 4.9 \\
\hline 0.70 & 4.7 & 4.7 & 5.4 & 0.9 & 1.7 & 6.1 & 2.4 & 1.0 & 3.1 & 4.3 & 4.9 & 7.4 \\
\hline 0.87 & 5.5 & 5.5 & 5.1 & 3.3 & 2.2 & 7.9 & 3.7 & 1.2 & 3.7 & 6.1 & 6.0 & 9.2 \\
\hline 1.05 & 5.7 & 5.7 & 3.6 & 7.7 & 2.6 & 9.3 & 5.2 & 1.9 & 4.1 & 8.4 & 6.4 & 10.7 \\
\hline 1.22 & 3.1 & 3.1 & 1.9 & 13.7 & 2.9 & 10.3 & 7.1 & 2.9 & 4.6 & 11.2 & 6.1 & 12.0 \\
\hline 1.40 & 3.2 & 3.2 & 0.6 & 20.1 & 3.3 & 10.6 & 7.4 & 3.3 & 4.7 & 13.7 & 5.2 & 12.9 \\
\hline 1.57 & 3.5 & 3.5 & 0.2 & 25.7 & 3.4 & 8.9 & 6.9 & 4.8 & 4.8 & 15.1 & 3.2 & 13.1 \\
\hline 1.75 & 3.1 & 3.1 & 1.0 & 29.9 & 3.2 & 7.2 & 6.3 & 6.8 & 5.0 & 11.5 & 1.8 & 12.5 \\
\hline 1.92 & 3.3 & 3.3 & 3.2 & 32.9 & 2.4 & 5.6 & 5.4 & 8.6 & 5.4 & 10.2 & 0.8 & 10.9 \\
\hline 2.09 & 4.0 & 4.0 & 6.5 & 32.8 & 1.8 & 4.5 & 4.4 & 8.6 & 6.5 & 8.6 & 0.5 & 9.0 \\
\hline 2.27 & 4.9 & 4.9 & 10.7 & 16.0 & 1.9 & 4.1 & 3.7 & 7.3 & 8.3 & 6.8 & 0.9 & 6.9 \\
\hline 2.44 & 2.4 & 2.4 & 15.5 & 10.4 & 2.9 & 1.7 & 3.1 & 6.0 & 4.8 & 5.1 & 2.1 & - \\
\hline 2.62 & 0.8 & 0.8 & 21.2 & 5.7 & 4.6 & 0.8 & 2.2 & 5.4 & 4.1 & 2.3 & 3.3 & 3.0 \\
\hline 2.79 & 0.1 & 0.1 & 1.0 & 2.4 & 6.6 & 0.3 & 1.0 & 5.4 & 3.8 & 0.3 & 4.3 & 1.6 \\
\hline 2.97 & 0.0 & 0.0 & 0.2 & 1.4 & 8.4 & 0.0 & 0.4 & 6.0 & 3.5 & -0.3 & 4.5 & 0.5 \\
\hline 3.14 & 0.5 & 0.5 & 1.3 & 2.9 & 9.7 & 0.3 & 0.0 & 6.9 & 3.0 & 0.1 & 4.0 & 0.0 \\
\hline 3.32 & 1.2 & 1.2 & 4.2 & 1.4 & 10.8 & 0.9 & 0.2 & 8.2 & 2.2 & 0.7 & 3.0 & 0.5 \\
\hline 3.49 & 1.9 & 1.9 & 8.4 & 2.4 & 7.8 & 1.5 & 0.8 & 10.2 & 1.8 & 2.7 & 1.7 & 1.6 \\
\hline 3.67 & 0.6 & 0.6 & 13.2 & 5.7 & 9.4 & 2.1 & 1.5 & 6.3 & 1.4 & 4.9 & 0.7 & 3.0 \\
\hline 3.84 & 0.8 & 0.8 & 17.9 & 10.4 & 11.6 & 3.1 & 2.4 & 3.8 & 1.4 & 6.9 & -0.1 & - \\
\hline 4.01 & 1.1 & 1.1 & 22.5 & 16.0 & 13.9 & 4.5 & 3.7 & 2.5 & 1.9 & 8.0 & -0.3 & 6.9 \\
\hline 4.19 & 1.4 & 1.4 & 26.9 & 32.8 & - & 6.2 & 5.2 & 1.9 & 3.3 & 8.5 & 0.8 & 9.0 \\
\hline 4.36 & 1.5 & 1.5 & 31.0 & 32.9 & 8.6 & 7.9 & 7.1 & 1.9 & 5.4 & 4.4 & 3.0 & 10.9 \\
\hline 4.54 & 1.9 & 1.9 & 33.0 & 29.9 & 6.4 & 9.2 & 7.4 & 2.7 & 7.8 & 4.7 & 6.0 & 12.5 \\
\hline 4.71 & 2.4 & 2.4 & 33.7 & 25.7 & 4.6 & 8.9 & 6.9 & 3.5 & 10.0 & 9.3 & 9.2 & 13.1 \\
\hline 4.89 & 2.5 & 2.5 & 1.4 & 20.1 & 3.5 & 4.9 & 6.3 & 2.8 & 11.9 & 6.8 & 11.7 & 12.9 \\
\hline 5.06 & 0.6 & 0.6 & 0.2 & 13.7 & 2.9 & 4.2 & 5.4 & 2.4 & 3.9 & 5.3 & 12.9 & 12.0 \\
\hline 5.24 & 0.9 & 0.9 & 0.9 & 7.7 & 2.7 & 4.0 & 4.4 & 2.4 & 3.6 & 4.7 & 12.5 & 10.7 \\
\hline 5.41 & 1.5 & 1.5 & 2.9 & 3.3 & 2.5 & 3.0 & 3.7 & 2.1 & 3.1 & 3.8 & 10.5 & 9.2 \\
\hline 5.59 & 2.0 & 2.0 & 5.1 & 0.9 & 1.9 & 2.3 & 3.1 & 1.7 & 1.6 & 3.2 & 7.7 & 7.4 \\
\hline 5.76 & 1.5 & 1.5 & 7.4 & 6.5 & 1.3 & 1.6 & 2.2 & 1.1 & 0.6 & 2.8 & 4.7 & 4.9 \\
\hline 5.93 & 0.9 & 0.9 & 1.2 & 3.2 & 0.8 & 0.8 & 1.0 & 0.9 & 0.6 & 1.4 & 2.2 & 2.2 \\
\hline 6.11 & 0.2 & 0.2 & 0.3 & 0.9 & 0.3 & 0.3 & 0.4 & - & - & 0.4 & 0.5 & 0.5 \\
\hline 6.28 & 0.0 & 0.0 & 0.0 & 0.0 & 0.0 & 0.1 & 0.0 & 0.0 & 0.0 & 0.0 & 0.0 & 0.0 \\
\hline
\end{tabular}

${ }^{b}$ Note that these two rotational potentials for radical 10 drop below zero for some rotational angles. This reflects the fact minimum energy conformations for all species were identified at the $\mathrm{HF} / 6-31 \mathrm{G}(\mathrm{d})$ level of theory, while final geometry optimizations and rotational scans were performed at the B3-LYP/6-31G(d) level. In this one case, the lowest energy conformation at the HF/6-31G(d) level is $0.3 \mathrm{~kJ}^{\mathrm{mol}}{ }^{-1}$ above the lowest-energy conformation at the B3-LYP/6-31G(d) level. 
Table S2 continued

\begin{tabular}{|c|c|c|c|c|c|c|c|c|c|c|c|c|}
\hline$\theta(\mathrm{rad})$ & $11 \mathrm{~b}$ & $11 \mathrm{c}$ & $11 d$ & $11 \mathrm{e}$ & $11 \mathrm{f}$ & $12 b$ & $12 d$ & $12 e$ & $17 \mathrm{~b}$ & $18 \mathrm{~b}$ & $19 b$ & $19 \mathrm{c}$ \\
\hline 0.00 & 0.0 & 0.0 & 0.0 & 0.0 & 0.0 & 0.7 & 2.7 & 0.0 & 0.0 & 0.0 & 0.0 & 0.0 \\
\hline 0.17 & 0.3 & 0.2 & 0.7 & 0.9 & 0.5 & 1.0 & 3.0 & 0.3 & 1.5 & 1.5 & 1.5 & 0.2 \\
\hline 0.35 & 1.5 & 0.6 & 2.1 & 3.7 & 2.0 & 2.1 & 3.4 & 1.4 & 6.0 & 5.9 & 6.0 & 1.2 \\
\hline 0.52 & 3.3 & 0.9 & 3.6 & 8.0 & 4.3 & 3.9 & 4.7 & 1.8 & 13.0 & 12.7 & 12.9 & 2.7 \\
\hline 0.70 & 4.4 & 1.0 & 4.8 & 13.2 & 6.7 & 6.5 & 4.9 & 2.7 & 21.7 & 21.2 & 21.3 & 4.0 \\
\hline 0.87 & 4.9 & 0.9 & 5.9 & 18.2 & 7.8 & 9.4 & 6.1 & 4.7 & 31.4 & 30.3 & 30.7 & 5.3 \\
\hline 1.05 & 5.5 & 0.8 & 6.5 & 21.9 & 10.0 & 12.9 & 7.6 & 6.6 & 41.0 & 39.0 & 39.9 & 6.3 \\
\hline 1.22 & 6.2 & 0.7 & 6.8 & 23.4 & 12.1 & 16.2 & 8.7 & 7.7 & 49.4 & 46.1 & 47.5 & 7.1 \\
\hline 1.40 & 7.2 & 0.7 & 7.0 & 23.1 & 13.1 & 18.1 & 10.4 & 7.9 & 55.2 & 50.0 & 53.1 & 7.6 \\
\hline 1.57 & 8.4 & 0.8 & 7.0 & 20.7 & 13.6 & 17.4 & 13.0 & 7.8 & 57.4 & 49.8 & 56.8 & 7.7 \\
\hline 1.75 & 9.7 & 0.6 & 5.1 & 17.7 & 13.9 & 12.8 & 15.9 & 7.3 & 56.4 & 46.8 & 58.1 & 7.2 \\
\hline 1.92 & 10.0 & 0.2 & 4.0 & 12.9 & 13.5 & 8.6 & 16.1 & 6.8 & 53.1 & 42.0 & 56.4 & 6.5 \\
\hline 2.09 & 10.8 & 0.0 & 3.5 & 10.1 & 12.7 & 5.0 & 15.1 & 6.5 & 47.8 & 36.5 & 50.8 & 5.5 \\
\hline 2.27 & 10.4 & 0.2 & 3.2 & 8.1 & 11.4 & 2.4 & 13.7 & 6.8 & 40.6 & 31.4 & 43.5 & 4.4 \\
\hline 2.44 & 9.9 & 0.6 & 3.1 & 7.3 & 10.0 & 0.7 & 11.7 & 7.7 & 33.1 & 27.5 & 35.8 & 3.2 \\
\hline 2.62 & 8.0 & 0.9 & 3.1 & 8.1 & 8.6 & 0.0 & 9.1 & 9.4 & 26.5 & 24.9 & 29.1 & 1.8 \\
\hline 2.79 & 6.0 & 1.0 & 3.1 & 9.7 & 7.3 & 0.0 & 6.0 & 11.5 & 21.5 & 24.4 & 23.8 & 0.6 \\
\hline 2.97 & 4.5 & 0.9 & 3.4 & 11.7 & 6.3 & 1.0 & 3.0 & 13.6 & 18.5 & 25.7 & 20.3 & 0.1 \\
\hline 3.14 & 3.7 & 0.8 & 3.4 & 13.5 & 5.9 & 2.7 & 0.7 & 14.9 & 17.6 & 28.7 & 18.8 & 0.1 \\
\hline 3.32 & 3.4 & 0.7 & 3.7 & 14.8 & 6.2 & 4.1 & 0.1 & 15.0 & 18.9 & 24.4 & 19.9 & 0.6 \\
\hline 3.49 & 3.3 & 0.7 & 4.8 & 15.5 & 7.1 & 5.6 & 0.0 & 13.9 & 22.3 & 24.9 & 23.5 & 1.4 \\
\hline 3.67 & 3.2 & 0.8 & 5.5 & 15.6 & 8.3 & 7.7 & 1.5 & 12.4 & 27.6 & 27.5 & 29.3 & 2.0 \\
\hline 3.84 & 3.0 & 0.6 & 6.4 & 15.6 & 9.6 & 10.0 & 4.4 & 11.2 & 34.4 & 31.4 & 36.7 & 2.4 \\
\hline 4.01 & 3.1 & 0.2 & 8.2 & 15.5 & 10.7 & 12.5 & 8.0 & 10.3 & 42.0 & 36.5 & 44.3 & 2.5 \\
\hline 4.19 & 3.4 & 0.0 & 10.9 & 15.5 & 11.6 & 15.1 & 11.2 & 9.8 & 48.9 & 42.0 & 50.1 & 3.0 \\
\hline 4.36 & 4.0 & 0.2 & 13.4 & 15.2 & 11.5 & 17.3 & 15.4 & 9.6 & 53.9 & 46.8 & 54.4 & 3.4 \\
\hline 4.54 & 5.0 & 0.6 & 15.0 & 14.6 & 11.0 & 18.9 & 20.1 & 10.0 & 56.8 & 49.9 & 56.9 & 3.7 \\
\hline 4.71 & 6.0 & 0.9 & 14.3 & 14.2 & 10.3 & 18.8 & 24.5 & 10.9 & 57.2 & 49.9 & 56.8 & 3.7 \\
\hline 4.89 & 6.5 & 1.0 & 12.0 & 14.1 & 9.6 & 6.2 & 21.9 & 12.2 & 54.4 & 46.0 & 53.4 & 3.5 \\
\hline 5.06 & 6.9 & 0.9 & 5.8 & 14.0 & 9.2 & 5.0 & 21.3 & 12.9 & 48.1 & 38.9 & 46.7 & 3.0 \\
\hline 5.24 & 7.0 & 0.8 & 8.6 & 13.2 & 8.8 & 3.2 & 20.1 & 12.7 & 39.3 & 30.1 & 37.9 & 2.6 \\
\hline 5.41 & 6.8 & 0.7 & 7.5 & 11.1 & 6.0 & 2.5 & 18.0 & 11.2 & 29.6 & 21.0 & 28.4 & 2.4 \\
\hline 5.59 & 2.1 & 0.7 & 6.5 & 8.8 & 4.7 & 2.6 & 14.8 & 8.7 & 20.1 & 12.6 & 19.3 & 2.2 \\
\hline 5.76 & 1.0 & 0.8 & 5.3 & 7.5 & 3.2 & 1.1 & 6.6 & 5.6 & 11.6 & 5.8 & 11.5 & 1.6 \\
\hline 5.93 & 0.6 & 0.6 & 2.9 & 7.5 & 1.7 & 1.0 & 3.8 & 2.7 & 5.0 & 1.5 & 5.6 & 1.0 \\
\hline 6.11 & 0.4 & 0.2 & 0.6 & - & 0.6 & 0.9 & 2.4 & 0.7 & 1.0 & 0.0 & 1.5 & 0.3 \\
\hline 6.28 & 0.0 & 0.0 & 0.0 & 0.0 & 0.0 & 0.7 & - & 0.0 & 0.0 & 1.5 & 0.0 & 0.0 \\
\hline
\end{tabular}


Table S3. Rotational barriers $\left(\mathrm{kJ} \mathrm{mol}^{-1}\right)$, Symmetry Numbers ( $\left.\sigma\right)$, Reduced Moments of

Inertia $\left(\mathrm{I}_{\mathrm{r}}\right)$ and Associated Enthalpy $\left(\mathrm{H}, \mathrm{kJ} \mathrm{mol}^{-1}\right)$ and Entropy $\left(\mathrm{S}, \mathrm{J} \mathrm{mol}^{-1} \mathrm{~K}^{-1}\right)$ at $333.15 \mathrm{~K}$

for the Modes Treated as Hindered Internal Rotations ${ }^{a}$

\begin{tabular}{|c|c|c|c|c|c|c|c|}
\hline \multirow[t]{2}{*}{ Mode $^{b}$} & \multirow[t]{2}{*}{ Barrier } & \multirow[t]{2}{*}{$\sigma$} & \multirow[t]{2}{*}{$\operatorname{lr}$} & \multicolumn{2}{|c|}{ Hindered Rotor } & \multicolumn{2}{|c|}{ Harmonic Oscillator } \\
\hline & & & & $\mathrm{H}$ & $S$ & $\mathrm{H}$ & $s$ \\
\hline $1 a$ & 3.9 & 3 & 2.59 & 1.9 & 13.8 & 2.0 & 12.0 \\
\hline $1 \mathrm{~b}$ & See Table S2 & 1 & 30.43 & 2.7 & 18.8 & 2.2 & 15.0 \\
\hline $1 \mathrm{c}$ & 1.3 & 3 & 3.12 & 1.6 & 15.3 & 2.3 & 16.4 \\
\hline $1 d$ & 0.6 & 3 & 3.13 & 1.4 & 15.4 & 2.4 & 19.9 \\
\hline $2 a$ & 4.9 & 3 & 2.91 & 2.1 & 13.8 & 1.9 & 11.1 \\
\hline $2 b$ & See Table S2 & 1 & 21.84 & 2.5 & 19.2 & 2.3 & 16.2 \\
\hline $2 c$ & See Table S2 & 2 & 69.31 & 2.5 & 27.1 & 2.5 & 22.6 \\
\hline $2 d$ & 0.3 & 3 & 2.94 & 1.4 & 15.2 & 2.6 & 24.7 \\
\hline 3a & 3.7 & 3 & 2.74 & 1.9 & 14.1 & 2.0 & 12.0 \\
\hline $3 b$ & See Table S2 & 1 & 26.35 & 2.4 & 17.4 & 2.2 & 15.2 \\
\hline $3 c$ & See Table S2 & 1 & 112.29 & 3.0 & 34.1 & 2.6 & 25.8 \\
\hline $3 d$ & See Table S2 & 2 & 86.61 & 3.0 & 28.4 & 2.5 & 21.1 \\
\hline $3 e$ & 0.8 & 3 & 2.64 & 1.5 & 14.7 & 2.4 & 18.3 \\
\hline $4 a$ & 7.3 & 3 & 2.43 & 2.0 & 11.9 & 1.7 & 9.7 \\
\hline $4 b$ & See Table S2 & 1 & 35.37 & 2.4 & 16.6 & 2.1 & 13.7 \\
\hline $4 c$ & 3.0 & 3 & 2.81 & 1.9 & 14.5 & 2.0 & 12.2 \\
\hline $4 d$ & See Table S2 & 1 & 101.01 & 4.0 & 33.6 & 2.6 & 24.3 \\
\hline $4 e$ & See Table S2 & 1 & 14.16 & 2.0 & 12.5 & 2.3 & 16.2 \\
\hline $4 f$ & 3.7 & 3 & 2.62 & 1.9 & 13.9 & 2.0 & 12.4 \\
\hline $5 a$ & 4.6 & 3 & 2.13 & 1.9 & 12.7 & 1.9 & 10.9 \\
\hline $5 b$ & See Table S2 & 1 & 35.96 & 2.6 & 18.7 & 2.1 & 14.0 \\
\hline $5 c$ & 1.0 & 3 & 2.83 & 1.5 & 15.0 & 2.3 & 16.7 \\
\hline $5 d$ & See Table S2 & 1 & 124.47 & 2.9 & 39.1 & 2.7 & 29.6 \\
\hline $6 a$ & 6.3 & 3 & 2.26 & 2.0 & 12.1 & 1.8 & 10.7 \\
\hline $6 b$ & See Table S2 & 1 & 35.15 & 2.8 & 18.9 & 2.1 & 13.9 \\
\hline $6 c$ & 0.6 & 3 & 2.92 & 1.4 & 15.1 & 2.3 & 17.4 \\
\hline $6 d$ & See Table S2 & 1 & 101.30 & 3.6 & 33.9 & 2.7 & 28.9 \\
\hline $6 e$ & 10.7 & 3 & 2.53 & 1.9 & 10.4 & 1.8 & 10.0 \\
\hline $6 f$ & 9.2 & 3 & 2.84 & 2.0 & 11.5 & 1.8 & 10.2 \\
\hline $7 a$ & 4.4 & 3 & 2.63 & 2.0 & 13.7 & 2.1 & 13.4 \\
\hline $7 \mathrm{~b}$ & See Table S2 & 2 & 28.02 & 2.5 & 27.0 & 2.5 & 20.9 \\
\hline $7 \mathrm{c}$ & 2.0 & 3 & 3.12 & 1.7 & 15.2 & 2.2 & 14.9 \\
\hline $7 d$ & See Table S2 & 2 & 21.56 & 2.4 & 25.9 & 2.5 & 23.3 \\
\hline $7 \mathrm{e}$ & 5.3 & 3 & 2.90 & 2.1 & 13.6 & 2.0 & 12.3 \\
\hline
\end{tabular}

${ }^{a}$ Rotational barriers calculated at the B3-LYP/6-31G(d) level of theory. Barriers are provided for those modes for which the rotational potential could be approximated as a simple cosine potential; for the other modes, full rotational potentials are provided in Table S2.

${ }^{b}$ See Figures $\mathrm{S} 1-3$. 
Table S3 continued

\begin{tabular}{|c|c|c|c|c|c|c|c|}
\hline \multirow[t]{2}{*}{ Mode } & \multirow[t]{2}{*}{ Barrier } & \multirow[t]{2}{*}{$\sigma$} & \multirow[t]{2}{*}{$\mathrm{I}_{\mathrm{r}}$} & \multicolumn{2}{|c|}{ Hindered Rotor } & \multicolumn{2}{|c|}{ Harmonic Oscillator } \\
\hline & & & & $\mathrm{H}$ & S & & \\
\hline $8 a$ & 4.2 & 3 & 2.82 & 2.0 & 14.0 & $2.0^{c}$ & $12.7^{c}$ \\
\hline $8 b$ & See Table S2 & 1 & 25.83 & 2.5 & 28.7 & $2.3^{c}$ & $17.9^{c}$ \\
\hline $8 c$ & See Table S2 & 1 & 66.76 & 3.0 & 30.7 & 2.4 & 20.0 \\
\hline $9 a$ & 5.3 & 3 & 2.32 & 2.0 & 12.7 & 2.0 & 13.0 \\
\hline $9 b$ & See Table S2 & 1 & 37.97 & 3.1 & 32.1 & 2.3 & 17.6 \\
\hline $9 c$ & See Table S2 & 1 & 101.21 & 2.9 & 35.9 & 2.5 & 21.8 \\
\hline $9 d$ & See Table S2 & 2 & 81.04 & 2.9 & 29.9 & 2.7 & 29.2 \\
\hline $9 e$ & See Table S2 & 1 & 24.29 & 3.0 & 30.7 & 2.5 & 23.3 \\
\hline $9 f$ & 3.5 & 3 & 2.85 & 1.9 & 14.3 & 2.0 & 12.7 \\
\hline $10 a$ & 3.6 & 3 & 2.61 & 1.9 & 13.9 & 2.0 & 11.9 \\
\hline $10 b$ & See Table S2 & 1 & 29.50 & 3.2 & 31.8 & 2.4 & 19.9 \\
\hline $10 c$ & 1.7 & 3 & 2.53 & 1.6 & 14.4 & 2.3 & 16.7 \\
\hline $10 d$ & See Table S2 & 1 & 97.03 & 3.2 & 34.7 & 2.4 & 19.0 \\
\hline $10 e$ & See Table S2 & 1 & 96.08 & 3.1 & 35.9 & 2.6 & 27.6 \\
\hline $10 f$ & See Table S2 & 2 & 77.20 & 3.0 & 26.3 & 2.6 & 25.1 \\
\hline $11 a$ & 9.2 & 3 & 2.39 & 2.0 & 10.9 & 1.8 & 10.1 \\
\hline $11 b$ & See Table S2 & 1 & 34.28 & 3.6 & 31.0 & 2.3 & 16.9 \\
\hline $11 \mathrm{c}$ & See Table S2 & 3 & 2.79 & 1.5 & 14.9 & 2.2 & 14.4 \\
\hline $11 d$ & See Table S2 & 1 & 87.20 & 4.2 & 34.8 & 2.6 & 25.3 \\
\hline $11 \mathrm{e}$ & See Table S2 & 1 & 61.08 & 3.7 & 23.9 & 2.4 & 19.6 \\
\hline $11 f$ & See Table S2 & 1 & 34.88 & 3.8 & 27.1 & 2.5 & 22.0 \\
\hline $11 \mathrm{~g}$ & 3.1 & 3 & 2.74 & 1.9 & 14.3 & 2.0 & 12.3 \\
\hline $12 a$ & 6.8 & 3 & 2.92 & 2.1 & 12.9 & 1.8 & 10.2 \\
\hline $12 b$ & See Table S2 & 1 & 19.13 & 2.7 & 27.1 & 2.5 & 21.8 \\
\hline $12 \mathrm{c}$ & 2.1 & 3 & 2.86 & 1.7 & 14.8 & 2.3 & 16.7 \\
\hline $12 d$ & See Table S2 & 1 & 64.17 & 3.4 & 29.7 & 2.4 & 17.9 \\
\hline $12 \mathrm{e}$ & See Table S2 & 1 & 84.21 & 3.7 & 30.6 & 2.6 & 26.5 \\
\hline $12 f$ & 16.2 & 3 & 2.44 & 1.6 & 8.1 & 1.5 & 7.6 \\
\hline $12 \mathrm{~g}$ & 14.2 & 3 & 3.03 & 1.8 & 9.6 & 1.6 & 8.4 \\
\hline $15 a$ & 2.6 & 3 & 3.02 & 1.8 & 14.9 & 2.3 & 16.3 \\
\hline $16 a$ & 3.5 & 3 & 2.80 & 1.9 & 14.3 & 2.1 & 13.3 \\
\hline $17 a$ & 3.9 & 3 & 2.74 & 2.0 & 14.0 & 2.0 & 12.1 \\
\hline $17 \mathrm{~b}$ & See Table S2 & 1 & 24.78 & 2.2 & 15.4 & 2.0 & 12.9 \\
\hline $17 \mathrm{c}$ & 0.6 & 3 & 3.11 & 1.4 & 15.4 & 2.6 & 23.5 \\
\hline $18 a$ & 5.1 & 3 & 2.95 & 2.1 & 13.8 & 1.9 & 11.3 \\
\hline $18 b$ & See Table S2 & 1 & 18.34 & 2.1 & 15.7 & 2.3 & 16.4 \\
\hline $18 \mathrm{c}$ & 13.6 & 2 & 57.58 & 2.8 & 18.6 & 2.5 & 21.4 \\
\hline $19 a$ & 4.3 & 3 & 2.83 & 2.0 & 14.0 & 2.0 & 11.9 \\
\hline $19 b$ & See Table S2 & 1 & 22.84 & 2.2 & 14.9 & 2.1 & 13.8 \\
\hline $19 c$ & See Table S2 & 1 & 83.69 & 2.9 & 36.3 & 2.7 & 30.5 \\
\hline $19 d$ & 8.4 & 2 & 72.67 & 2.9 & 18.4 & 2.5 & 22.0 \\
\hline
\end{tabular}

${ }^{c}$ Under the harmonic oscillator model, the pairs of modes corresponding to each of $8 \mathrm{a}$ and $8 \mathrm{~b}$ are non-equivalent owing to coupling (which is not considered in the one-dimensional hindered rotor treatment). The average harmonic oscillator values are thus reported here; the individual values are $\mathrm{H}=2.0$ and 2.0 and $\mathrm{S}=13.0$ and 12.5 (for $8 \mathrm{a}$ ), and $\mathrm{H}=2.5$ and 2.2 and $\mathrm{S}=20.2$ and 15.5 (for $8 \mathrm{~b}$ ). 
Table S4. Enthalpy, Entropy and Gibb's Free Energy Changes Along the Minimum

Energy Path for the Addition Reactions $\mathrm{R} \bullet+\mathrm{S}=\mathrm{C}(\mathrm{Z}) \mathrm{SCH}_{3}$ at 333.15K

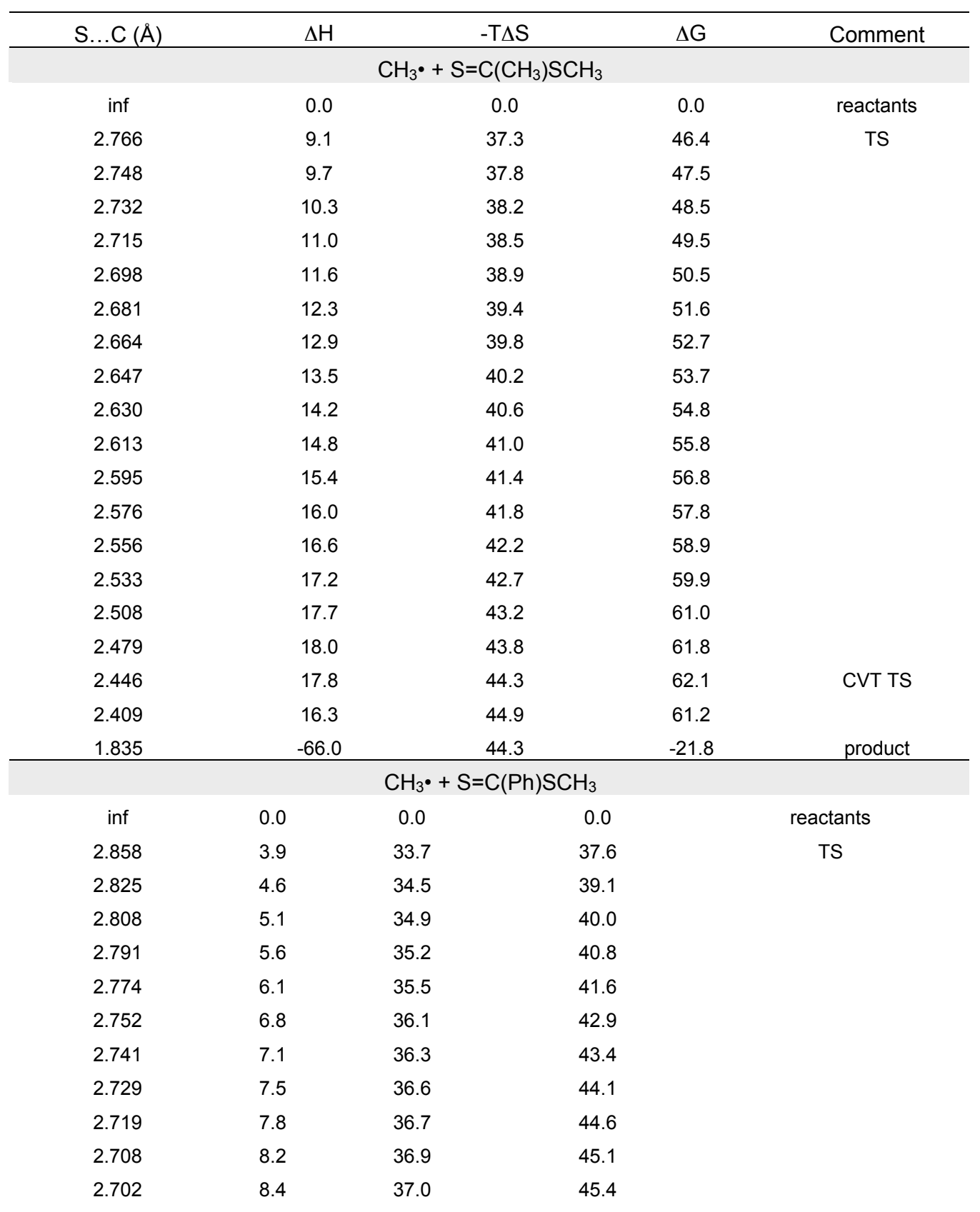

${ }^{a}$ IRCs calculated at the B3-LYP/6-31G(d) level of theory. At each geometry, $\Delta \mathrm{H}$ was calculated using improved energies obtained at the RMP2/6-311+G(3df,2p) level of theory, while $\Delta \mathrm{S}$ (and also temperature correction to $\Delta \mathrm{H}$ ) were calculated using the B3-LYP/6-31G(d) geometries and frequencies in conjunction with the harmonic oscillator approximation. Note that, for the sake of clarity, the energies are plotted as a function of the forming bond length. However, they correspond to points on the minimum energy path of the reaction, which was calculated as a function of the intrinsic reaction coordinate (in mass weighted units). 


\section{Table S4 continued}

\begin{tabular}{|c|c|c|c|c|}
\hline S...C $(\AA)$ & $\Delta \mathrm{H}$ & $-\mathrm{T} \Delta \mathrm{S}$ & $\Delta G$ & Comment \\
\hline 2.695 & 8.6 & 37.1 & 45.7 & \\
\hline 2.688 & 8.8 & 37.3 & 46.1 & \\
\hline 2.671 & 9.4 & 37.6 & 46.9 & \\
\hline 2.654 & 9.9 & 37.8 & 47.7 & \\
\hline 2.637 & 10.4 & 38.1 & 48.5 & \\
\hline 2.620 & 10.9 & 38.3 & 49.2 & \\
\hline 2.603 & 11.4 & 38.6 & 49.9 & \\
\hline 2.586 & 11.8 & 38.8 & 50.6 & \\
\hline 2.568 & 12.2 & 39.0 & 51.1 & \\
\hline 2.498 & 11.0 & 41.7 & 52.7 & CVT TS \\
\hline 2.458 & 6.6 & 42.1 & 48.7 & \\
\hline 1.836 & -95.2 & 48.7 & -46.5 & product \\
\hline \multicolumn{5}{|c|}{$\mathrm{CH}_{3} \cdot+\mathrm{S}=\mathrm{C}\left(\mathrm{CH}_{2} \mathrm{Ph}\right) \mathrm{SCH}_{3}$} \\
\hline $\inf$ & 0.0 & 0.0 & 0.0 & reactants \\
\hline 2.784 & 3.3 & 40.1 & 43.4 & TS \\
\hline 2.767 & 3.7 & 40.4 & 44.1 & \\
\hline 2.750 & 4.2 & 40.8 & 45.0 & \\
\hline 2.734 & 4.8 & 41.2 & 45.9 & \\
\hline 2.717 & 5.3 & 41.6 & 46.9 & \\
\hline 2.700 & 5.9 & 41.9 & 47.8 & \\
\hline 2.683 & 6.4 & 42.3 & 48.7 & \\
\hline 2.666 & 7.0 & 42.7 & 49.7 & \\
\hline 2.649 & 7.6 & 43.0 & 50.6 & \\
\hline 2.632 & 8.1 & 43.4 & 51.5 & \\
\hline 2.615 & 8.6 & 43.7 & 52.4 & \\
\hline 2.598 & 9.1 & 44.1 & 53.2 & \\
\hline 2.580 & 9.6 & 44.4 & 54.0 & \\
\hline 2.563 & 10.1 & 44.7 & 54.8 & \\
\hline 2.546 & 10.4 & 45.0 & 55.5 & \\
\hline 2.529 & 10.7 & 45.3 & 56.1 & \\
\hline 2.512 & 10.9 & 45.6 & 56.6 & \\
\hline 2.495 & 11.0 & 45.9 & 56.9 & \\
\hline 2.483 & 11.0 & 46.1 & 57.1 & CVT TS \\
\hline 2.461 & 7.9 & 46.4 & 54.3 & \\
\hline 1.851 & -72.9 & 44.0 & -29.0 & product \\
\hline \multicolumn{5}{|c|}{$\cdot \mathrm{CH}_{2} \mathrm{COOCH}_{3}+\mathrm{S}=\mathrm{C}\left(\mathrm{CH}_{3}\right) \mathrm{SCH}_{3}$} \\
\hline $\inf$ & 0.0 & 0.0 & 0.0 & reactants \\
\hline 2.547 & -16.8 & 56.4 & 39.6 & TS \\
\hline 2.534 & -16.5 & 56.7 & 40.2 & \\
\hline 2.517 & -16.2 & 57.0 & 40.8 & \\
\hline 2.500 & -16.0 & 57.3 & 41.3 & \\
\hline 2.483 & -15.8 & 57.5 & 41.7 & \\
\hline 2.466 & -15.8 & 57.8 & 42.0 & \\
\hline 2.449 & -15.9 & 58.1 & 42.1 & CVT TS \\
\hline
\end{tabular}




\section{Table S4 continued}

\begin{tabular}{|c|c|c|c|c|}
\hline S...C $(\AA)$ & $\Delta \mathrm{H}$ & $-\mathrm{T} \Delta \mathrm{S}$ & $\Delta \mathrm{G}$ & Comment \\
\hline 2.432 & -16.3 & 58.3 & 42.0 & \\
\hline 2.415 & -16.9 & 58.5 & 41.7 & \\
\hline 2.413 & -16.9 & 58.6 & 41.6 & \\
\hline 2.411 & -17.0 & 58.6 & 41.6 & \\
\hline 2.410 & -17.1 & 58.6 & 41.5 & \\
\hline 2.408 & -17.2 & 58.6 & 41.4 & \\
\hline 2.406 & -17.3 & 58.7 & 41.4 & \\
\hline 2.405 & -17.4 & 58.7 & 41.3 & \\
\hline 2.403 & -17.5 & 58.7 & 41.2 & \\
\hline 2.401 & -17.6 & 58.7 & 41.2 & \\
\hline 2.400 & -17.7 & 58.7 & 41.1 & \\
\hline 2.398 & -17.8 & 58.8 & 41.0 & \\
\hline 2.396 & -17.9 & 58.8 & 40.9 & \\
\hline 2.394 & -18.0 & 58.8 & 40.8 & \\
\hline 2.393 & -18.1 & 58.8 & 40.7 & \\
\hline 2.391 & -18.3 & 58.8 & 40.6 & \\
\hline 2.389 & -18.4 & 58.9 & 40.5 & \\
\hline 2.388 & -18.5 & 58.9 & 40.4 & \\
\hline 2.386 & -18.7 & 58.9 & 40.2 & \\
\hline 2.384 & -18.8 & 58.9 & 40.1 & \\
\hline 2.382 & -19.0 & 58.9 & 40.0 & \\
\hline 2.381 & -19.1 & 59.0 & 39.8 & \\
\hline 2.379 & -19.3 & 59.0 & 39.7 & \\
\hline 2.377 & -19.5 & 59.0 & 39.5 & \\
\hline 2.376 & -19.6 & 59.0 & 39.4 & \\
\hline 2.374 & -19.8 & 59.0 & 39.2 & \\
\hline 2.372 & -20.0 & 59.1 & 39.1 & \\
\hline 1.859 & -59.3 & 55.7 & -3.6 & product \\
\hline \multicolumn{5}{|c|}{ - $\mathrm{CH}_{2} \mathrm{Ph}+\mathrm{S}=\mathrm{C}\left(\mathrm{CH}_{3}\right) \mathrm{SCH}_{3}$} \\
\hline $\inf$ & 0.0 & 0.0 & 0.0 & \multirow[t]{8}{*}{ reactants } \\
\hline 2.552 & -9.1 & 47.5 & 38.4 & \\
\hline 2.538 & -8.8 & 48.1 & 39.4 & \\
\hline 2.522 & -8.4 & 48.8 & 40.3 & \\
\hline 2.506 & -8.2 & 49.3 & 41.1 & \\
\hline 2.489 & -8.0 & 49.7 & 41.8 & \\
\hline 2.473 & -7.9 & 50.2 & 42.2 & \\
\hline 2.457 & -8.0 & 50.6 & 42.5 & \\
\hline 2.436 & -8.5 & 51.0 & 42.5 & \multirow[t]{5}{*}{$\mathrm{CVT}=\mathrm{TS}$} \\
\hline 2.431 & -8.7 & 51.1 & 42.5 & \\
\hline 2.416 & -9.3 & 51.4 & 42.1 & \\
\hline 2.399 & -10.4 & 51.7 & 41.2 & \\
\hline 1.886 & -46.6 & 49.6 & 3.0 & \\
\hline \multicolumn{5}{|c|}{ - $\mathrm{C}\left(\mathrm{CH}_{3}\right)_{2} \mathrm{CN}+\mathrm{S}=\mathrm{C}\left(\mathrm{CH}_{3}\right) \mathrm{SCH}_{3}$} \\
\hline $\inf$ & 0.0 & 0.0 & 0.0 & \\
\hline
\end{tabular}




\section{Table $S 4$ continued}

\begin{tabular}{ccccc}
\hline $\mathrm{S} \ldots \mathrm{C}(\AA)$ & $\Delta \mathrm{H}$ & $-\mathrm{T} \Delta \mathrm{S}$ & $\Delta \mathrm{G}$ & Comment \\
\hline 2.525 & -11.7 & 58.5 & 46.8 & \\
2.510 & -11.2 & 58.7 & 47.5 & \\
2.525 & -11.7 & 58.5 & 46.8 & \\
2.478 & -10.5 & 59.2 & 48.7 & \\
2.462 & -10.3 & 59.4 & 49.2 & \\
2.446 & -10.2 & 59.7 & 49.5 & \\
2.430 & -10.3 & 59.9 & 49.7 & CVT TS \\
2.414 & -10.5 & 60.2 & 49.7 & \\
2.398 & -11.0 & 60.4 & 49.4 & \\
2.382 & -11.9 & 60.7 & 48.8 & TS \\
2.363 & -13.4 & 61.0 & 47.5 & product \\
2.358 & -14.0 & 61.1 & 47.1 & \\
1.913 & -41.0 & 61.7 & 20.6 & \\
& & & &
\end{tabular}


Figure S1. Variationally Optimized B3-LYP/6-31G(d) Geometries of the Transition

Structures for $\mathrm{R} \bullet+\mathrm{S}=\mathrm{C}(\mathrm{Z}) \mathrm{SCH}_{3}$, for $\mathrm{R}=\mathrm{CH}_{3}$ with $\mathrm{Z}=\mathrm{CH}_{3}, \mathrm{Ph}$ and $\mathrm{CH}_{2} \mathrm{PH}$ and $\mathrm{Z}=\mathrm{CH}_{3}$ with $\mathrm{R}=\mathrm{CH}_{2} \mathrm{COOCH3}, \mathrm{CH}_{2} \mathrm{Ph}$ and $\mathrm{C}\left(\mathrm{CH}_{3}\right)_{2} \mathrm{CN}$, Showing the Modes Treated As Hindered Internal Rotations

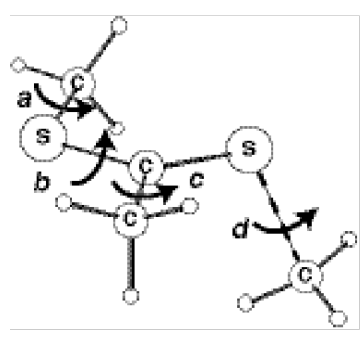

1

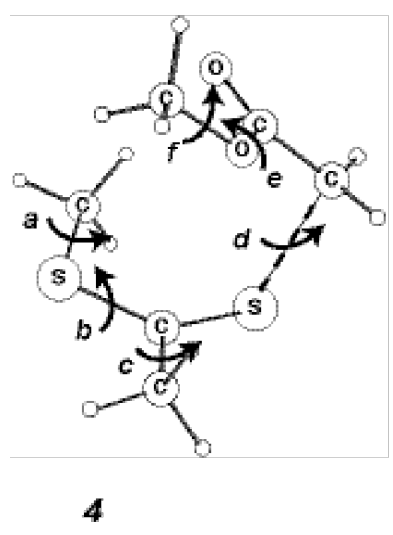

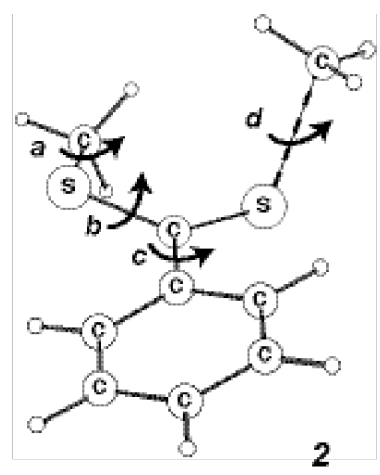

2

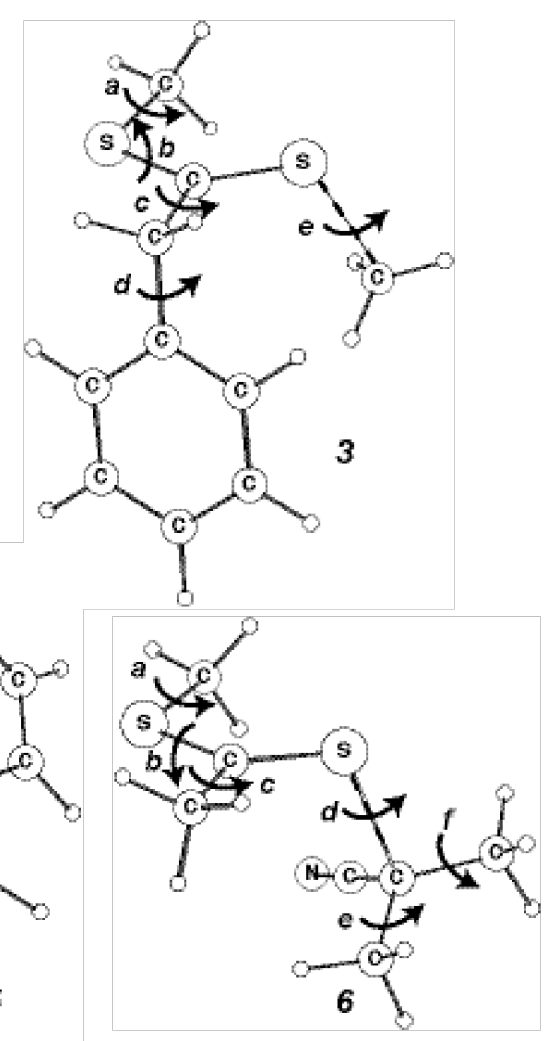

S15 
Figure S2. Optimized B3-LYP/6-31G(d) Geometries of the RAFT-Adduct Radicals $\mathrm{RSC} \cdot(\mathrm{Z}) \mathrm{SCH}_{3}$, for $\mathrm{R}=\mathrm{CH}_{3}$ with $\mathrm{Z}=\mathrm{CH}_{3}, \mathrm{Ph}$ and $\mathrm{CH}_{2} \mathrm{PH}$ and $\mathrm{Z}=\mathrm{CH}_{3}$ with $\mathrm{R}=$ $\mathrm{CH}_{2} \mathrm{COOCH}, \mathrm{CH}_{2} \mathrm{Ph}$ and $\mathrm{C}\left(\mathrm{CH}_{3}\right)_{2} \mathrm{CN}$, Showing the Modes Treated As Hindered Internal Rotations

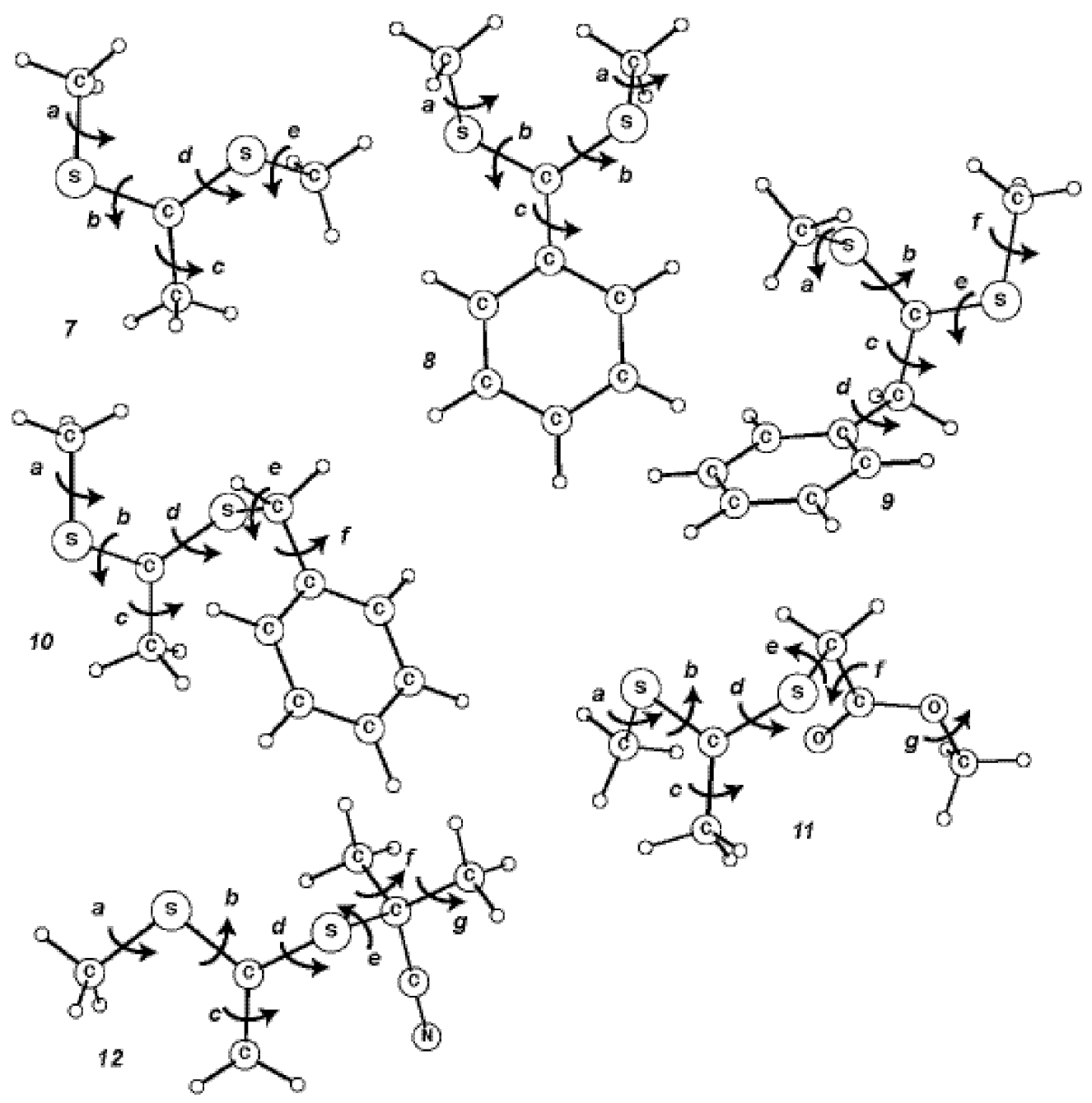


Figure S3. Optimized B3-LYP/6-31G(d) Geometries of the Reactants in $\mathrm{R} \bullet+\mathrm{S}=\mathrm{C}(\mathrm{Z}) \mathrm{SCH}_{3}$, for $\mathrm{R}=\mathrm{CH}_{3}$ with $\mathrm{Z}=\mathrm{CH}_{3}$, Ph and $\mathrm{CH}_{2} \mathrm{PH}$ and $\mathrm{Z}=\mathrm{CH}_{3}$ with $\mathrm{R}=\mathrm{CH}_{2} \mathrm{COOCH}, \mathrm{CH}_{2} \mathrm{Ph}$ and $\mathrm{C}\left(\mathrm{CH}_{3}\right)_{2} \mathrm{CN}$, Showing the Modes Treated As Hindered Internal Rotations
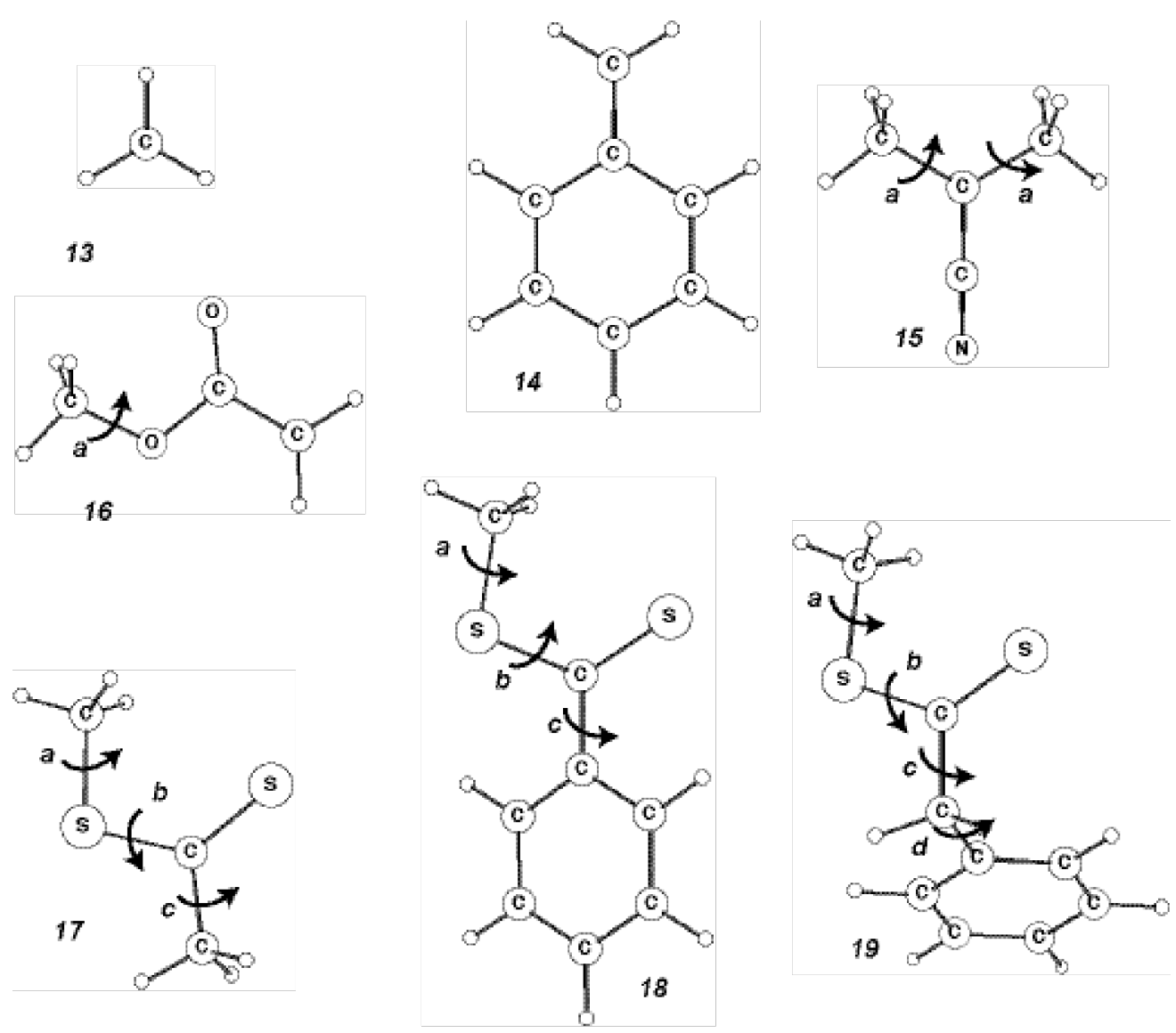
Figure S4. Enthalpy, Entropy (as -T $\Delta S$ ), and Gibb's Free Energy $\left(\mathrm{kJ} \mathrm{mol}^{-1}\right)$ versus S---C

\section{Bond Length (̊̊) Along Minimum Energy Path of the Addition Reactions (1)-(6).}
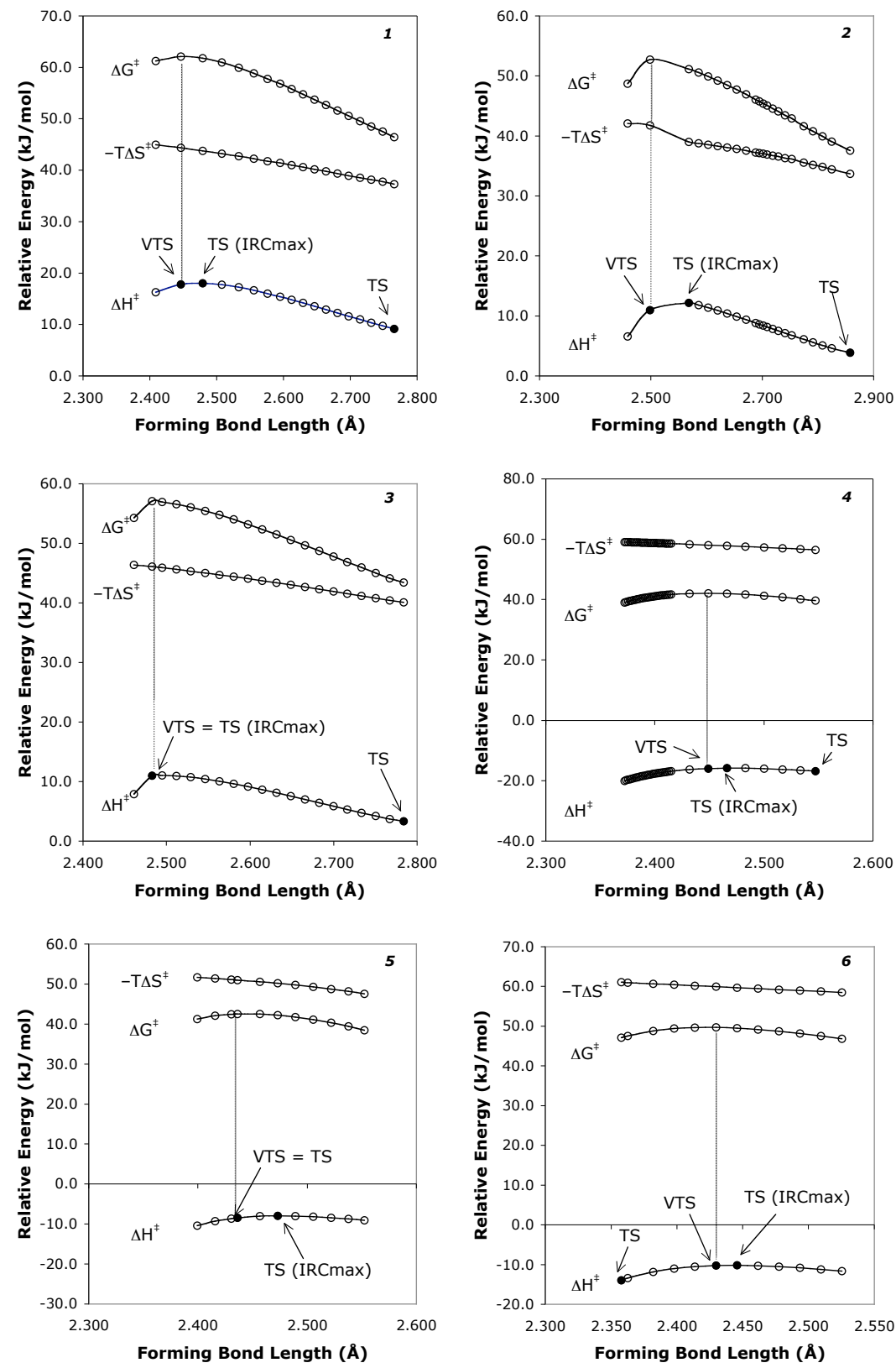

\footnotetext{
* Note that, for the sake of clarity, the energies are plotted as a function of the forming bond length. However, they correspond to points on the minimum energy path of the reaction, which was calculated as a function of the intrinsic reaction coordinate (in mass weighted units).
} 